\title{
EASI RBD-FAST: an efficient method of global sensitivity analysis for present and future challenges in building performance simulation
}

\author{
Jeanne Goffart ${ }^{1}$, Monika Woloszyn \\ LOCIE, Univ. Savoie Mont Blanc, CNRS, Chambéry, 73000 France
}

\begin{abstract}
In the field of building performance simulation, there is a growing interest for the use of sensitivity analysis (SA) and uncertainty analyses (UA), that enable to estimate the uncertainty in model prediction and to identify which model inputs are mainly responsible for this uncertainty. However, several methods exist, with different capabilities and limitations, leading to some misuse. The objective and the first novelty of the present study was to conduct a structured and comprehensive comparison of the capabilities of two different methods: EASI RBD-FAST (a variance-based computation of Sobol indices) and the popular Morris screening. This comparison was made on two annual outputs representative of winter (heating demand) and summer (overheating) from a comprehensive detached house energy model. It was shown that both methods fulfil basic expectations, since the same clusters of influential parameters were identified with the same computation effort. We have also shown that the EASI RBD-FAST method allows, with the same number of simulations, to extract very relevant additional information: intuitive sensitivity index and uncertainty of the output. Moreover, a novel application to compute temporal sensitivity indices on time-series outputs (such as free-floating temperature) was proposed. It demonstrated the possibility of using SA to investigate the dynamic properties of a building, such as persistence or inertia. In addition, the EASI RBD-FAST method illustrated in this paper proves to be not only very useful but also easy to use and accessible tool for building performance simulations.
\end{abstract}

Keywords: uncertainty and sensitivity analysis, time-dependent output, ANOVA, Morris method, building energy modelling

Highlights:

- sensitivity and uncertainty analysis (SA/UA) are powerful supports of building performance simulation

- EASI RBD-FAST (ANOVA method) and Morris screening were applied on a low energy house

- both methods indicated the most influential parameters on heating demand and summer overheating

- EASI RBD-FAST enables both SA, UA and temporal sensitivity indices computations with limited cost.

- EASI RBD-FAST has been recently implemented in SALib in Python - it is now easy to use

\footnotetext{
${ }^{1}$ Corresponding author: jeanne.goffart@gmail.com 


\section{Introduction}

In the field of building performance, the use of numerical models and simulation tools is a very active research and application domain. In the literature, many papers are devoted to the enhancement of physical models, improving some specific character, reducing the gap between the simulated results and the measurements, while expanding model complexity. The drawback is that more complex models require additional and more detailed information, which may be unknown or highly uncertain. The input approximation, or "guess", introduced then by the modeler can affect significantly model results. As stated by Saltelli et al. [1] "It is therefore essential to understand the impact of these uncertainties on the model output, if the model is to be used effectively and responsibly in any decision-making process. Sensitivity analysis (SA) and uncertainty analysis (UA) are the two main tools used in exploring the uncertainty of such models."

Sensitivity analysis can be defined on one hand as the study of how the uncertainty in model results (outputs) can be explained and quantified by the uncertainty of the model inputs (parameters and/or boundary conditions). Uncertainty analysis on the other hand aims to estimate the uncertainty in model prediction without identifying which model input is responsible. Thus, both are related but distinct approaches. Some methods, as the variance-based methods, aim to combine both uncertainty and sensitivity analysis to optimize the extractable information from model output variability. This specific point is detailed further in this work.

There is a wide range of applications of SA/UA. For example, they help identifying assumptions primarily responsible for output variations, and then prioritize research to model or measure the key input to reduce output uncertainty. They are also very useful to find main parameters needed to construct a simplified model, by indicating the non-influential inputs that can be disregarded in the simplified model. Finally, they are often used to gain confidence in model results by constructing robust simulations, including uncertainty and consequently confidence bound. In the case of building simulation we can cite many studies, as for example about energy performance guarantee for an office building [2], extraction of the most influential Input selection for Bayesian calibration of an office building [3], or a dwelling [4], an early design input exploration for decision making [5], [6] or a comparison between simulation and in situ measurement [7], [8].

There are many very different SA methods [9], some of them enabling also uncertainty quantification. In general, they are grouped into two main categories. The first one is referred to as "Local sensitivity analysis" (LSA) and can be used if the input parameter variability is close to the nominal value, as a "perturbation". Local approaches are based on derivatives and are suitable for linear models. If however the input parameter variability concerns a significant range of variability, methods from the second category, called "global sensitivity analysis" (GSA) should be used [10]. Global sensitivity analysis involves simultaneous variation of several inputs to catch possible interactions among the inputs through the model, unlike the so-called One-at-the-Time (OAT) methods, that belong to the local approach category. Both categories include several different methods.

For a specific problem, the choice of a SA method is based on the model complexity, computational time, desired extractable information and, last but not least, the usability and accessibility of the algorithm [10] [1] [11]. Consequently, a large variability of SA tools is used in different fields such as chemistry, economics, mathematics, medicine, biology and physics [12]. 
More specifically, SA tools have been more and more used for the last two decades in buildings physics, as presented in thorough reviews by Tian in [13] and more recently by Pang et al. in [14].

Some difficulties arise with high fidelity Building Performance Simulation (BPS) as defined by Petersen et al. [15], because models are non-linear, non-monotonic and often discontinuous, thus nondifferentiable [16], [17]. In such cases, only global approaches are adapted for valid and henceforth reliable sensitivity analyses $[9,10]$. Indeed, local/OAT approach is not recommended, as suggested by Andrea and Annoni in [18], because up to $99 \%$ of the inputs space may be unexplored and lead to erroneous conclusions.

However, because the nonlinearities in BPS are often considered as minor, numerous studies report the use of local/OAT approaches. Their extensive use is mainly due to the ease of implementation and the low computational cost. Yet, several review studies report as much as $42 \%$ [1], of un-adapted use of SA methods in different fields, failing to properly explore the space of the input factors. In engineering and energy fields, this misuse reaches half of highly cited papers. The same authors point out the urgent and important need of developing good practices.

To allow good practice in SA for building performance simulation, global sensitivity approach has to be considered, among them those based on the ANalysis Of Variance (ANOVA) that compute the intuitive Sobol first order sensitivity index (SI) [19]. This variance-based sensitivity measure quantifies the part of output variability due to input variability and provides a value between 0 and 1 . For instance, an input with a SI equal to 0.75 is responsible for $75 \%$ of the output variability.

The estimation of the sensitivity indices using ANOVA methods is robust (as long as the number of samples is sufficient), appropriated for the BPS complexity, their interpretation is intuitive; and the information on the sensitivity and the uncertainty is extracted using the same set of simulations. However, ANOVA methods are often associated with prohibitive calculation times ranging from several thousand to hundreds of thousands simulations, especially with the original Sobol method $[13-15,20]$.

This explains the development of the popular screening Morris method in BPS, considered as a good compromise between reliability, usability and computational cost $[14,15]$. Several comparisons between Sobol and Morris methods show indeed a simulation cost divided by 100 and sometimes 1000 [15]. In all cases, the method identifies main influential input cluster [21] and must be used for preliminary simulations before uncertainty analyses or model simplifications $[22,23]$. However, unlike variance-based methods, the Morris result is only qualitative and sometimes the rank obtained is unstable, especially for non-linear behaviour $[15,20,21]$. In addition, Morris method does not allow uncertainty analysis, which is an important step of SA. Indeed," before uncertainty can be apportioned it needs to be estimated" [1]

As sensitivity analyses is an abundant research field, the SA research community has developed new methods and improvements which make it possible to enhance sensitivity estimates convergence and so to drastically reduce the cost of calculation such as sampling design optimizations (Lptau, LHS, etc.), bias reduction and calculation schemes. Depending on their accessibility (practical implementation) these improvements will be adopted or not by the BPS community. For example, for the method of Morris, Campolongo et al. [24] developed a space exploration generation method which divides the number of simulations by 10 to obtain a stable result. Of the 16 Morris usage studies cited by Petersen et al. [15], only two, [2,25], use it. Thus the implementation in easily accessible tools and awareness of 
advances in sensitivity analysis are the keys to the adoption of good practices in building physics and more generally in other fields of applications $[1,11]$.

Similarly, algorithms enabling to compute Sobol indices were improved as well. For example, firstorder SI calculation scheme with permutation trick which, combined with efficient sampling design in Sobol method, leads to compute all sensitivity first order indexes with only two matrices. This calculation scheme previously introduced by Mara and Rakoto [26] and used in BPS by Goffart et al. in $[27,28]$, reduces the number of simulations at $2 * 1000$ simulations instead of $8 * 1000$ simulations needed by the classic approach for 6 inputs [29].

Specifically, the Fourier amplitude sensitivity test (FAST) is improved by the random balanced design (RBD) technique [30]. The RBD-FAST method combined with a specific first-order SI calculation scheme named EASI [31] to compute all sensitivity first order indices with one sample set, seems very promising. The EASI RBD-FAST method reduces the computational burden by several orders of magnitude. Papers using this calculation scheme in BPS as [32,33], show the algorithm capabilities. For example, Goffart et al. [33] estimate the sensitivity indices for 14 inputs in a highly non-linear hygrothermal BPS with less of 200 simulations according to the convergence result.

\section{Scope of the paper}

As stated above, many studies from the literature confirm that global Sensitivity and Uncertainty Analysis (SA/UA) can be powerful supports of building performance simulation. However, their use is not always straightforward. A recent study by Saltelli et al. [1] emphasizes that in engineering and energy fields, an un-adapted use of SA methods reaches half of highly cited papers. The authors of this study point out the urgent and important need of developing good practices. The ambition of the present paper is to address this question and to contribute to the development of good practices of SA/UA in building performance simulation.

Consequently, the main objective of the present study is to raise awareness on a highly informative and accessible variance-based sensitivity analysis method. More specifically, the RBDFAST, coupled with EASI first order SI calculation scheme, is explained and applied. The novelty is twofold. First, a structured, step-by-step explanation and method comparison is conducted and discussed on a case-study from building performance simulation. Second, a global sensitivity analysis on time series output is proposed, as an innovative application of SA.

To this purpose, a comparison is made between the popular Morris screening method and the EASI RBD-FAST method, for performance simulations of an energy efficient detached house. In order to compare both methods on similar bases for computational effort, the same number of simulations (a few hundreds) will be performed with the same uncertain 38 inputs taken from boundary conditions, building management and material thermophysical properties. To explore linear and non-linear response models, the sensitivity analyses are made first for aggregated winter and summer indicators with respectively heating energy demand and degree-days of summer discomfort. Then, from the same set of simulation, the paper shows how additional information, such as uncertainty analysis and trends, can be estimated with EASI RBD-FAST. The final part of the paper deepens the information extraction using the EASI RBD-FAST sensitivity analysis of a temporal output, illustrated by the internal operative temperature output for building overheating issues. 
The immediately subsequent section 2 describes in detail the Morris and the EASI RBD-FAST methods, followed by a description of the case study that served for the numerical application. Then, section 3 presents the comparison between both methods, and illustrates how additional and valuable information can be extracted from the simulations set from the EASI RBD-FAST method. Finally, the perspective use of SA on temporal outputs is detailed in section 4.

\section{Sensitivity Analysis on BPS by Morris and EASI RBD-FAST}

\subsection{Comparative description of Morris and EASI RBD-FAST methods}

To better understand the differences between the variance-based EASI RBD-FAST method and the Morris screening method, a summary diagram is shown in Figure 1 . The diagram is divided into the three main steps of a sensitivity method. The first is the sampling design, which has an impact on the filling of the exploration space and thus on the variability of the inputs propagating in the model. The second step is the sensitivity estimation, which is the calculation procedure for assessing the impact of input variability on output variability. The final step is the sensitivity measure or measure of importance, which represents the indicator for the interpretation of the sensitivity results. In the description of the methods below, reference is made to this summary diagram to better illustrate the point.

\subsubsection{Morris screening: An improved "One at the time " method}

The sampling design of the Morris method is a space discretization by mesh and node as illustrated in Figure 1. The principle of the mesh is the following: the range of variation of the k parameters is discretized in $Q$ levels, the crossing of these $Q$ levels defines a set of nodes $Q^{k}$. The Morris method consists in sampling these nodes by a set of $r$ random trajectories each of which passes through $k+1$ nodes so that each factor varies only once per trajectory. The number of simulations depends on the number $k$ of parameters considered in the analysis and the number of paths $r$ such as $N=r(k+1)$. On the Figure 1 an example is made with $\mathrm{k}=2$ inputs for $Q=4$ levels and $r=3$ trajectories, that induces $\mathrm{N}=9$ samples.

The principle of the Morris method is a one parameter at a time (OAT) sensitivity estimation through the global space thanks to the trajectory, repeated in several points of the parameter space. The method combines the speed of typical OAT methods while exploring a mesh on a global scale and thus limits the risk of missing areas of the parameters space. No area of the space can be favoured, so Morris's method allows only uniform probability distribution estimation over an initial range of 0 to 1 , which is then transposed to the ranges of expected variations.

The influence of each factor $X_{i}$ is evaluated by comparing two simulations of a trajectory among which only the factor $X_{i}$ has varied. This is the elementary effect, i.e. the proper effect of $X_{i}$ that is estimated at a point in space. This analysis for each trajectory makes it possible to extract the global influence of $X_{i}$ on the output by the mean of the absolute value of the elementary effects $\left(\mu^{*}\right)$ and the variability of the effects by the standard deviation $(\sigma)$. The latter qualifies a level of interaction and / or nonlinearity of the parameter in the model. The sensitivity measure is extracted from a graphical representation, named Morris graph, of the global effect $\left(\mu^{*}\right)$ and the interaction or non-linearity level $(\sigma)$ as illustrated in Figure 1 . The mean of the absolute value of elementary effects $\left(\mu^{*}\right)$ allows to classify the parameters in different groups and to visualize their placement relative to each other. Three groups of inputs are generally identified as: the input(s) with no effect; the input(s) with linear effect and the input(s) with non-linear effect or/and with interactions (see Figure 1). 
The information extracted by the Morris method remains qualitative and only a hierarchy of effects is possible. Only the points belonging to the multidimensional grid constructed by the method can be evaluated, the extracted information is therefore dependent on the mesh set up and the number of trajectories. Values of levels number $Q>=4$ and trajectories number $r$ close to 100 are appropriate for BPS purpose [15]. In the present study a value of $Q=4$ is chosen, and 80 trajectories are performed but only 8 are kept for model evaluation. Indeed, to reduce the number of simulations induced by trajectories, a sampling design improvement developed by Campolongo et al. [24] is used. This algorithm improves the space filling by selecting one in ten trajectory that best estimate the space exploration.

\subsubsection{Estimating the Sobol sensitivity indices with the RBD FAST Method}

Sobol sensitivity indices are the most popular measures of importance. These indices are based on the complete decomposition of the model's output variability $V$ into a sum of fractional variances: Such a decomposition is unique, if and only if, the random variables are independent [19]

$$
\begin{aligned}
& V=\sum_{i} V_{i}+\sum_{i<j} V_{i j}+\sum_{i<j<m} V_{i j m}+\cdots+V_{12 \ldots k} \\
& 1=\sum_{i} S_{i}+\sum_{i<j} S_{i j}+\sum_{i<j<m} S_{i j m}+\cdots+S_{12 \ldots k}
\end{aligned}
$$

where $S_{i}=V_{i} / V$ is the first-order sensitivity index that measures the amount of the response variance induced by the disturbed input $X_{i}$ alone. The other indexes, $S_{i j}, S_{i j m}, \ldots$ are sensitivity indices of order 2 to $k$, which measure the amount of the response variance explained by input interactions in the model. . The indices are between 0 and 1. A high index indicates a strong relationship between the variation of $X_{i}$ and that of the output $Y$, it is the sensitivity measure computed by EASI RBD-FAST and represented in Figure 1 . The total sum of first-order sensitivity indices significantly less than 1 implies interactions between parameters.

There are many numerical methods proposed in the literature to assess the variance-based sensitivity indices. The Sobol design method [34] uses the principle of comparing the output variances between two sample matrices by fixing only one input between the two matrices, which requires a large number of evaluations. Other methods use the direct estimation of indices by decomposing the variance by projection on a basis. The FAST (Fourier Amplitude Sensitivity Test) method uses a frequency basis [35] while the principle of the chaos polynomial expansion methods uses a polynomial basis [36]. For the last method called 'metamodel' there are other approaches and therefore other bases such as Gaussian processes [37] .

Whatever the approach considered, many developments and improvements have been made to optimize convergence, i.e. the accuracy of the sensitivity estimator while reducing the number of evaluations of the model and therefore the cost of the calculation. In the Sobol design methods the accuracy depends on the number of evaluations $\mathrm{N}$ and the number of parameters $k$. Only RBD-FAST (Random Balance Design - Fourier Amplitude Sensitivity Test) based methods and the metamodel approach allow the estimation of the sensitivity indices with only $\mathrm{N}$ evaluations of the model, independently from the number of input parameters k. However, the metamodel approaches first involve validation of the metamodel and, only then, using it for sensitivity analysis. This procedure is not straightforward and complicates the accessibility of the method. 
The main advantage of the RBD-FAST method [33] is the estimation of robust and accurate first-order Sobol indices with only a set of $\mathrm{N}$-simulations of the order of a few hundreds of evaluations, regardless of the number of parameters. To do so, the method evaluates the partial variance from the analysis of the first frequencies of the decomposition of $Y$ by Fourier transform. The originality of RBD-FAST is the use of randomization by permutation which brings forward a single and same frequency $\omega_{0}$ for each random variable $X_{i}$. The procedure is as follows: (1) random permutation $R_{i}$ of the set of samples for each input variable $X_{i}$ sampled beforehand with the same frequency $\omega_{0} ;(2)$ simulation of the model using permuted sets of input variables; (3) rearranging the $N$ output values according to the permutation vectors $R_{i}$ for each input variable $X_{i}$. For each rearranged set, the frequency $\omega_{0}$ is reconstructed for a single entry, the other entries remaining random. A frequency analysis of the $\mathrm{N}$ output $Y$ thus reorganized will show the effect of the input $X_{i}$ in the frequency $\omega_{0}$ and its harmonics $\left\{2 \omega_{0}, 4 \omega_{0}, 6 \omega_{0}, \cdots\right\}$. The effect of all the other variables will be a white noise spread over the whole range of frequencies. This procedure is the sensitivity estimation using RBD-FAST and is illustrated in Figure 1 for the case of a triangular-shaped vector with frequency $\omega_{0}$. To generalize this method, the Effective Algorithm for variance-based Sensitivity Indices (EASI) [31], a pre-processing algorithm, transforms a random sample of $\mathrm{N}$ simulations into a triangular waveform of frequency $\omega_{0}=1$. The EASI approach of RBD-FAST has the advantage that no specific sampling scheme is necessary, which makes it possible to use already available data obtained from a standard sampling method as Monte-Carlo or quasi Monte-Carlo methods. The disadvantage of the EASI RBD FAST method is that it computes the first-order terms only. To enhance the sensitivity estimates accuracy a bias correction developed by Tissot and Prieur [38] is considered in the method. To optimize convergence and minimize the number of model evaluations, an advanced Monte-Carlo sampling method is used in this work : LHS Maximin [39]. More information on RBD-FAST with EASI and its application in BPS is available in [33].

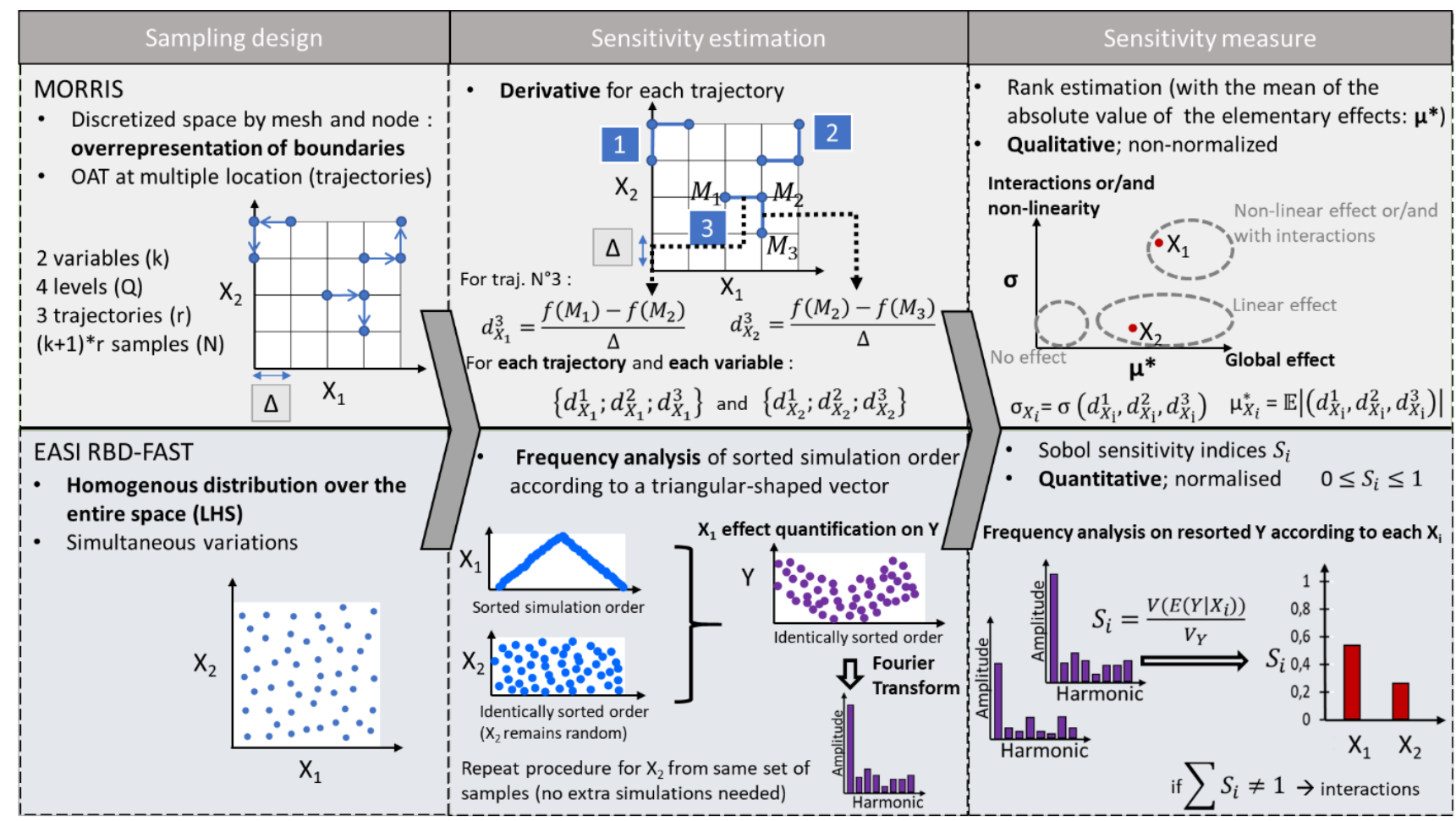

Figure 1: Comparative summary diagram of Morris method (upper side) and EASI RBD-FAST method (lower side) in terms of sampling design, sensitivity estimation and sensitivity measure 


\subsection{Case study specifications}

The objective of the study is primarily to compare the capabilities of EASI RBD-FAST and Morris screening on a coherent energy-efficient house model with many parameters for sensitivity analysis. Thus, the conclusions and approach of the study will focus on the performance of the respective methods rather than the interpretation and performance of the evaluated building. Nevertheless, the building energy model used for the study is based on a real detached house built as part of the COMEPOS project, a French national research program for the design, construction and monitoring of net zero energy detached houses [40]. The comparison between measurements and simulation results was performed and showed a consistent building simulation model [41].

\subsubsection{Numerical model}

The present case study, is an occupied house, located near Paris (France) and presented in Figure 2. As shown in Figure 3 the kitchen is open to the living room on the ground floor and three bedrooms are located on the first floor for a total living surface area of $80 \mathrm{~m}^{2}$. The house is well insulated with an inner insulation consisting of a layer of glass wool $\left(R=1.2 \mathrm{~m}^{2} . \mathrm{K} / \mathrm{W}\right)$ and a layer of polyurethane foam $\left(R=4.4 \mathrm{~m}^{2} . \mathrm{K} / \mathrm{W}\right)$. The floor is insulated with a layer of polyurethane foam $\left(\mathrm{R}=4.7 \mathrm{~m}^{2} . \mathrm{K} / \mathrm{W}\right)$ and the roof with glass wool $\left(\mathrm{R}=12.4 \mathrm{~m}^{2} . \mathrm{K} / \mathrm{W}\right)$. The house is south-north oriented. The windows are double-glazed. To limit the solar radiation entering the house during the summer period, roof overhangs of one meter deep have been installed above each window on the south façade. Moreover, each window is equipped with a roller shutter. The ventilation system is a balanced mechanical ventilation system with heat recovery and a by-pass. The fan speed of the ventilation system can be modulated by the residents: the minimum speed corresponds to the basic flow rate value of $90 \mathrm{~m} 3 / \mathrm{h}$ in accordance with French thermal regulations. The maximum speed corresponds to an air flow rate of $150 \mathrm{~m} 3 / \mathrm{h}$ for nighttime mechanical ventilation in summer. The intermediate speed gives a flow rate of $120 \mathrm{~m} 3 / \mathrm{h}$.

The case study is modelled with EnergyPlus [42]. The building energy model is composed of 10 thermal zones, 8 represented in Figure 3, complemented with the attic and the crawl space.
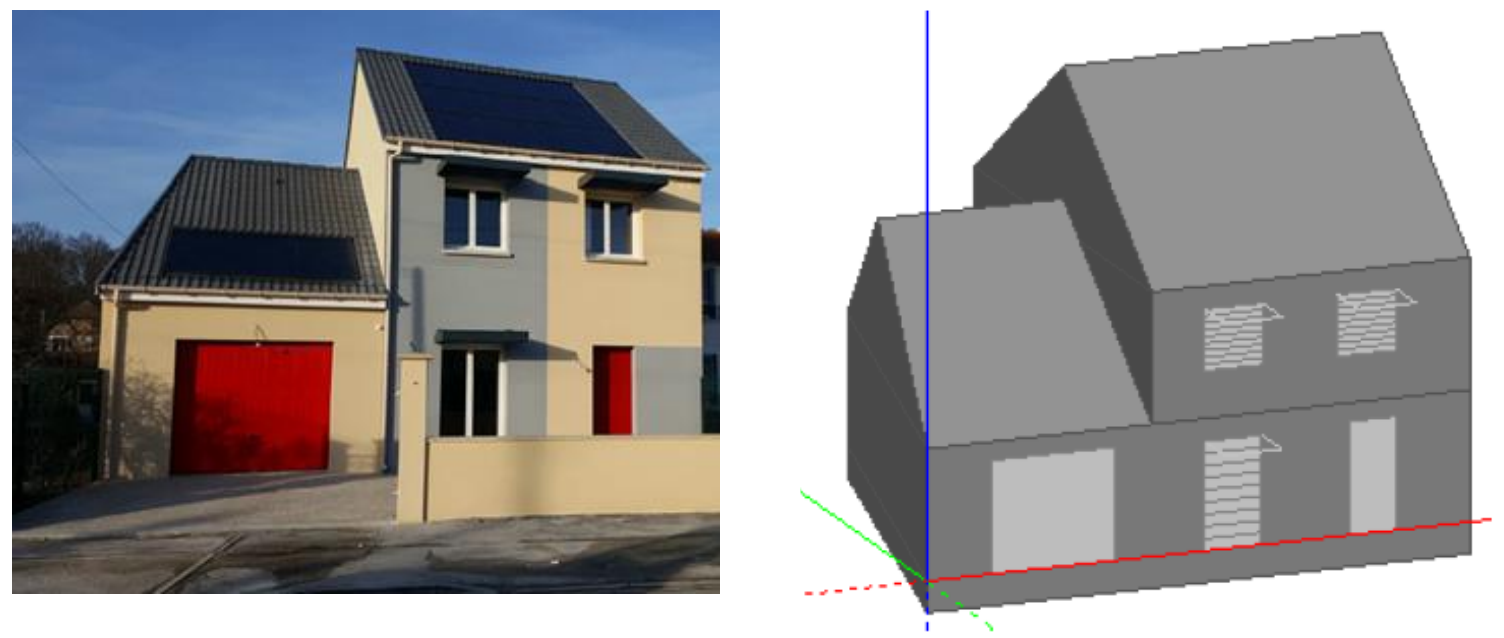

Figure 2: The building case study, on the left the south façade of the actual house which inspired the numerical model on the right 

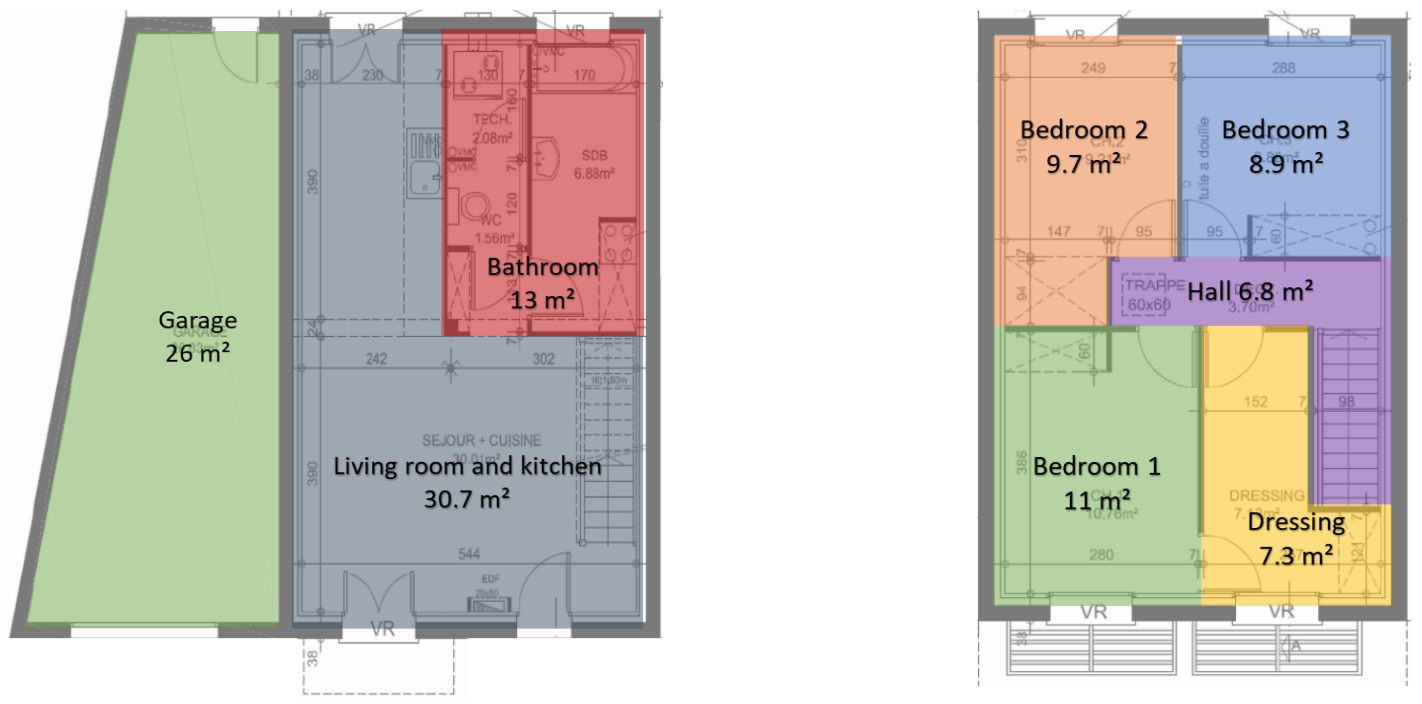

Figure 3: The building plan, the ground floor on the left, the first floor on the right

\subsubsection{Inputs and range of variation for the sensitivity analyses}

As discussed above, the main aim of the study is to illustrate the capabilities of EASI RBD-FAST and Morris for a typical large-dimensions building simulation case study for researchers and practitioners. To do so a comparison is made on key indicators between the two methods: the calculation time, the quality of the extracted information, the convergence of the results, but also the amount of information.

Two typical BPS outputs are selected as results of main interest: the annual heating need for winter behaviour and the risk of overheating for summer performance. The latter is expressed as degree.days of operative temperature over $27^{\circ} \mathrm{C}$ in the master bedroom (Bedroom 1), the room in the house that experienced the highest temperature episodes. The number of degrees strictly above $27{ }^{\circ} \mathrm{C}$ is calculated from the operating temperature over the year per hour and this result is converted into the number of days to obtain degree.day units. The input parameters considered in the sensitivity analysis are associated with the building, envelope properties, boundary conditions and building systems.

Thus, it is chosen to evaluate the impact of the selected input parameters variations on the results, using firstly the Morris method and secondly the EASI RBD-FAST method. Knowing that Morris' method imposes uniform probability distribution on inputs variability, it is chosen for all parameters to better compare both methods. Uniform probability distribution imposes event equiprobability between the possible values of the parameter, which is a strong constraint on the representation of the uncertainties. 
Table 1 : Main physical characteristics of the model with range of variation for the uncertain parameters for sensitivity analysis (in bold)

\begin{tabular}{|c|c|c|c|c|}
\hline Input parameters & $\begin{array}{l}\text { Nominal } \\
\text { value }\end{array}$ & Unit & $\begin{array}{l}\text { Relative } \\
\text { variation }\end{array}$ & $\begin{array}{l}\text { Probability } \\
\text { distribution }\end{array}$ \\
\hline \multicolumn{4}{|c|}{ Boundary conditions (4 inputs) } & \\
\hline $\begin{array}{l}\text { Occupant internal load during the } \\
\text { night }\end{array}$ & 63 & $\mathrm{~W}$ & $\pm 30 \%$ & Uniform \\
\hline Equipment internal load & 0.74 & $\mathrm{~W} / \mathrm{m}^{2}$ & $\pm 10 \%$ & Uniform \\
\hline Albedo & 0.3 & - & $\pm 33 \%$ & Uniform \\
\hline airtightness & $\begin{array}{c}\text { Room: } 0.03 \\
\text { Attics: } 0.5\end{array}$ & $\begin{array}{c}1 / \mathrm{hr} \\
\text { Air change per } \\
\text { hour }\end{array}$ & $\pm 20 \%$ & Uniform \\
\hline \multicolumn{4}{|c|}{ Building management (4 inputs) } & \\
\hline Air renewal in summer & 90 & $\mathrm{~m}^{3} / \mathrm{h}$ & $\pm 10 \%$ & Uniform \\
\hline Air renewal in winter & 90 & $\mathrm{~m}^{3} / \mathrm{h}$ & $\pm 10 \%$ & Uniform \\
\hline Heating setpoint temperature' & 20 & ${ }^{\circ} \mathrm{C}$ & $\pm 5 \%$ & Uniform \\
\hline Night mechanical ventilation rate & 120 & $\mathrm{~m}^{3} / \mathrm{h}$ & $\pm 20 \%$ & Uniform \\
\hline \multicolumn{4}{|c|}{ Thermophysical properties (30 inputs) } & \\
\hline \multicolumn{4}{|c|}{ Exterior wall } & \\
\hline $\begin{array}{l}\text { Concrete block } \\
\text { conductivity } \\
\text { thickness } \\
\text { density } \\
\text { specific heat }\end{array}$ & $\begin{array}{l}1.05 \\
0.20 \\
1300 \\
1.75 \\
\end{array}$ & $\begin{array}{c}\mathrm{W} /(\mathrm{m} \cdot \mathrm{K}) \\
\mathrm{m} \\
\mathrm{kg} / \mathrm{m}^{3} \\
\mathrm{~J} /(\mathrm{kg} \cdot \mathrm{K})\end{array}$ & $\pm 10 \%$ & Uniform \\
\hline \multicolumn{2}{|l|}{$\begin{array}{l}\text { Polyurethane* } \\
\text { conductivity } \\
\text { thickness } \\
\text { density } \\
\text { specific heat } \\
\text { *also involved in the attic floor with } \\
\text { a thickness of } 0.05 \mathrm{~m} \text { and in the low } \\
\text { floor }\end{array}$} & $\begin{array}{c}\mathrm{W} /(\mathrm{m} \cdot \mathrm{K}) \\
\mathrm{m} \\
\mathrm{kg} / \mathrm{m}^{3} \\
\mathrm{~J} /(\mathrm{kg} \cdot \mathrm{K})\end{array}$ & $\pm 10 \%$ & Uniform \\
\hline $\begin{array}{l}\text { Plasterboard* } \\
\text { conductivity } \\
\text { thickness } \\
\text { density } \\
\text { specific heat } \\
\text { *also involved in partition wall and } \\
\text { attic floor }\end{array}$ & $\begin{array}{c}0.26 \\
0.013 \\
825 \\
1008\end{array}$ & $\begin{array}{c}\mathrm{W} /(\mathrm{m} \cdot \mathrm{K}) \\
\mathrm{m} \\
\mathrm{kg} / \mathrm{m}^{3} \\
\mathrm{~J} /(\mathrm{kg} \cdot \mathrm{K})\end{array}$ & $\pm 10 \%$ & Uniform \\
\hline \multicolumn{4}{|c|}{ Intermediate floor } & \\
\hline $\begin{array}{l}\text { Concrete beam } \\
\text { conductivity } \\
\text { thickness } \\
\text { density } \\
\text { specific heat }\end{array}$ & $\begin{array}{c}1.23 \\
0.16 \\
1300 \\
648\end{array}$ & $\begin{array}{c}\mathrm{W} /(\mathrm{m} \cdot \mathrm{K}) \\
\mathrm{m} \\
\mathrm{kg} / \mathrm{m}^{3} \\
\mathrm{~J} /(\mathrm{kg} \cdot \mathrm{K})\end{array}$ & $\pm 10 \%$ & Uniform \\
\hline
\end{tabular}




\begin{tabular}{|c|c|c|c|c|}
\hline $\begin{array}{l}\text { Concrete screed* } \\
\text { conductivity } \\
\text { thickness } \\
\text { density } \\
\text { specific heat } \\
\text { *also involved in the low floor with } \\
\text { a thickness of } 0.05 \mathrm{~m}\end{array}$ & $\begin{array}{c}1.75 \\
0.08 \\
2400 \\
880\end{array}$ & $\begin{array}{c}\mathrm{W} /(\mathrm{m} \cdot \mathrm{K}) \\
\mathrm{m} \\
\mathrm{kg} / \mathrm{m}^{3} \\
\mathrm{~J} /(\mathrm{kg} \cdot \mathrm{K})\end{array}$ & $\pm 10 \%$ & Uniform \\
\hline $\begin{array}{l}\text { Concrete slab* } \\
\text { conductivity } \\
\text { thickness } \\
\text { density } \\
\text { specific heat } \\
\text { *also involved in the low floor with } \\
\text { a thickness of } 0.16 \mathrm{~m}\end{array}$ & $\begin{array}{l}1.75 \\
0.04 \\
2400 \\
1000\end{array}$ & $\begin{array}{c}\mathrm{W} /(\mathrm{m} \cdot \mathrm{K}) \\
\mathrm{m} \\
\mathrm{kg} / \mathrm{m}^{3} \\
\mathrm{~J} /(\mathrm{kg} \cdot \mathrm{K})\end{array}$ & $\pm 10 \%$ & Uniform \\
\hline $\begin{array}{l}\text { Tile floor } \\
\text { conductivity } \\
\text { thickness } \\
\text { density } \\
\text { specific heat }\end{array}$ & $\begin{array}{l}0.41 \\
0.01 \\
1200 \\
1000\end{array}$ & $\begin{array}{c}\mathrm{W} /(\mathrm{m} \cdot \mathrm{K}) \\
\mathrm{m} \\
\mathrm{kg} / \mathrm{m}^{3} \\
\mathrm{~J} /(\mathrm{kg} \cdot \mathrm{K})\end{array}$ & $\pm 10 \%$ & Uniform \\
\hline \multicolumn{4}{|c|}{ Partition wall } & \\
\hline \multicolumn{5}{|l|}{ Plasterboard (see exterior wall) } \\
\hline $\begin{array}{l}\text { Glass wool wall } \\
\text { conductivity } \\
\text { thickness } \\
\text { density } \\
\text { specific heat }\end{array}$ & $\begin{array}{c}0.038 \\
0.05 \\
18 \\
1030\end{array}$ & $\begin{array}{c}\mathrm{W} /(\mathrm{m} \cdot \mathrm{K}) \\
\mathrm{m} \\
\mathrm{kg} / \mathrm{m}^{3} \\
\mathrm{~J} /(\mathrm{kg} \cdot \mathrm{K})\end{array}$ & $\pm 10 \%$ & Uniform \\
\hline \multicolumn{5}{|l|}{ Plasterboard (see exterior wall) } \\
\hline \multicolumn{4}{|c|}{ Attic floor } & \\
\hline $\begin{array}{l}\text { Glass wool attic } \\
\text { conductivity } \\
\text { thickness } \\
\text { density } \\
\text { specific heat }\end{array}$ & $\begin{array}{c}0.032 \\
0.4 \\
18 \\
1030\end{array}$ & $\begin{array}{c}\mathrm{W} /(\mathrm{m} \cdot \mathrm{K}) \\
\mathrm{m} \\
\mathrm{kg} / \mathrm{m}^{3} \\
\mathrm{~J} /(\mathrm{kg} \cdot \mathrm{K})\end{array}$ & $\pm 10 \%$ & Uniform \\
\hline Polyurethane (see exterior wall) & & & & \\
\hline Plasterboard (see exterior wall) & & & & \\
\hline & indows & & & \\
\hline $\begin{array}{l}\text { U-factor } \\
\text { Solar heat gain coefficient } \\
\text { Visible transmittance }\end{array}$ & $\begin{array}{c}1.4 \\
0.37 \\
0.49\end{array}$ & $\begin{array}{c}\mathrm{W} / \mathrm{m} 2-\mathrm{K} \\
- \\
-\end{array}$ & $\pm 10 \%$ & Uniform \\
\hline
\end{tabular}

The choice of ranges of variation is important and is a delicate step of the sensitivity analysis. In the context of the study, the variations correspond to the operating phase of the building, they are reasonable with a maximum of $33 \%$ for the albedo and in agreement with the values of the physical quantities observed in literature, see the complete review of Tian et al. [43] on uncertainty analysis in building energy assessment for more detailed (Table 1 of their paper). 
In addition to the range of variation, the choice of inputs for the sensitivity analysis is essential. In the context of this study, many characteristics of the materials are considered in the analysis. However, the linear relationship between thickness and conductivity leads to a redundancy of information. So, depending on the material, either the thickness is considered uncertain or the conductivity is. In general, the conductivity is uncertain except for glass wool where the thickness is more difficult to manage due to its implementation and its soft character [44].

Finally, 38 parameters were considered in our sensitivity analysis. They are associated: to building uses, such as the internal loads of occupants during night $( \pm 10 \%)$; thermophysical parameters $( \pm 10 \%)$ such as conduction, specific heat and density of each building material as well as solar factor and thermal resistance of windows $( \pm 10 \%)$; boundary conditions such as albedo $(0.3 \pm 0.1)$, , infiltration $( \pm 20 \%)$ building management, such as ventilation ( $\pm 10 \%)$, set-point temperature for heating $\left( \pm 1^{\circ} \mathrm{C}\right)$, and the night mechanical ventilation rate $( \pm 20 \%)$. See details in the Table 1 .

\subsubsection{Statistical set up for sensitivity analyses}

The analysis is done with 312 simulations for each method. For Morris this corresponds to 8 trajectories of 39 simulations which corresponds to a nominal (reference) value plus 38 values for each parameter variation. These trajectories are previously optimized over 10 times more trajectories [24], as has been explained in section 2.1. The mesh is made with an usual and recommended value of $p=4$ [15]. 312 simulations have also been performed for the EASI RBD-FAST method, on the basis of a LHS Maximin sampling of the input parameters. To enable this study the Python sensitivity analysis library SALib [45] was used. In a further effort of accessibility and usability, the algorithm EASI RBD-FAST has been implemented in this Python SALib. The CPU (process) time for 312 simulations of the case study, generation of stochastic inputs and calculation of sensitivity measures is less than 3 hours, same for both methods. The results are exposed in the following section.

\section{Results of SA comparison on aggregated outputs}

\subsection{Convergence}

Before all, the first indicator to be verified is the convergence, i.e. the number of simulations sufficient to obtain stable sensitivity results. Figure 4 shows, for the heating energy demand and for both methods, the convergence of the mean and the variance of the statistical sample. The variance represents the variability of the model output and the mean the most likely output result. These are important statistical indicators for the SA using both methods.

The results show that starting from a low number of simulations, mean and variance take, regardless of the method used, very different values, which means that there can be no convergence of the SA yet. However, with a growing number of simulations, mean and variances reach a steady asymptotic value, meaning that the inputs space has been sufficiently explored to reach convergence and enable robust SA. Both methods reach convergence with 312 simulations that means that the sample is rich enough to perform valid sensitivity analyses of the model being evaluated. Interestingly, we can see that the variance and mean deviation for both methods are different: this is related to the difference in sampling method and especially due to the overrepresentation of the boundaries for Morris sampling procedure and is discussed further in the paper. 

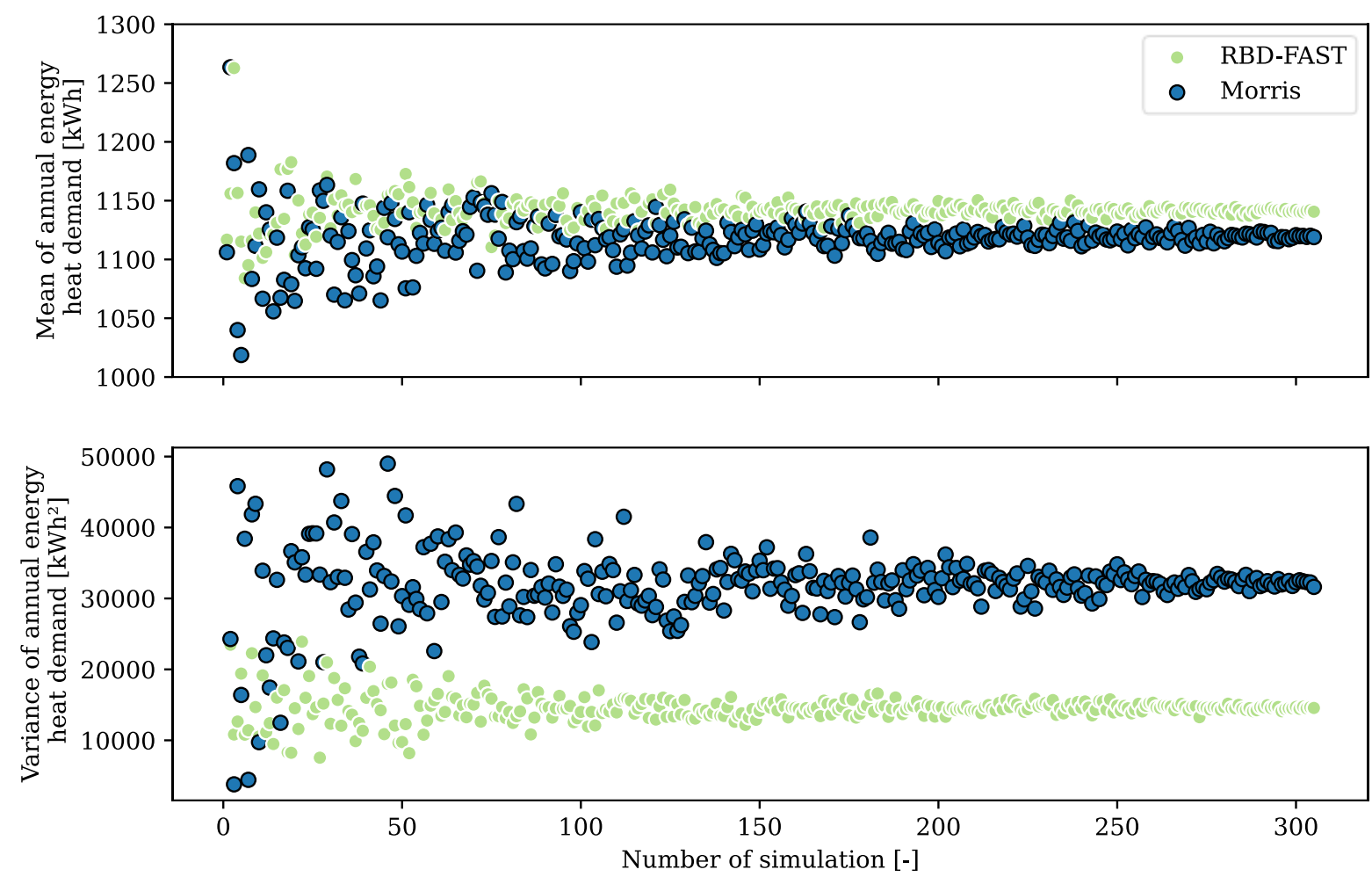

Figure 4: Variance and mean convergence for each method for energy heat demand ( $N=312$ )

\subsection{Sensitivity measure: part of variance versus rank}

\subsubsection{Winter behaviour: heating annual energy needs}

Depending on the method used, the sensitivity measures are quite different as has been shown in the summary diagram in Figure 1. As a reminder, in the case of EASI RBD-FAST, the sensitivity indices are calculated and quantify the variance part of each input parameter on the total variance i.e. the dispersion of the output. The indicator in the Morris method is only qualitative and allows to classify the parameters by their order of influence and to visualize their relative placement: their ranking. Figure 5 shows the graph of Morris method: one parameter is detached, it is the set-point temperature. Table 2 shows the parameters in descending order of influence according to EASI RBD-FAST, the sensitivity indices according to EASI RBD-FAST the sensitivity measures according to Morris ( $\mu^{*}$ et $\sigma$ ) and the rank obtained with the Morris's method according to the $\mu^{*}$ value. 


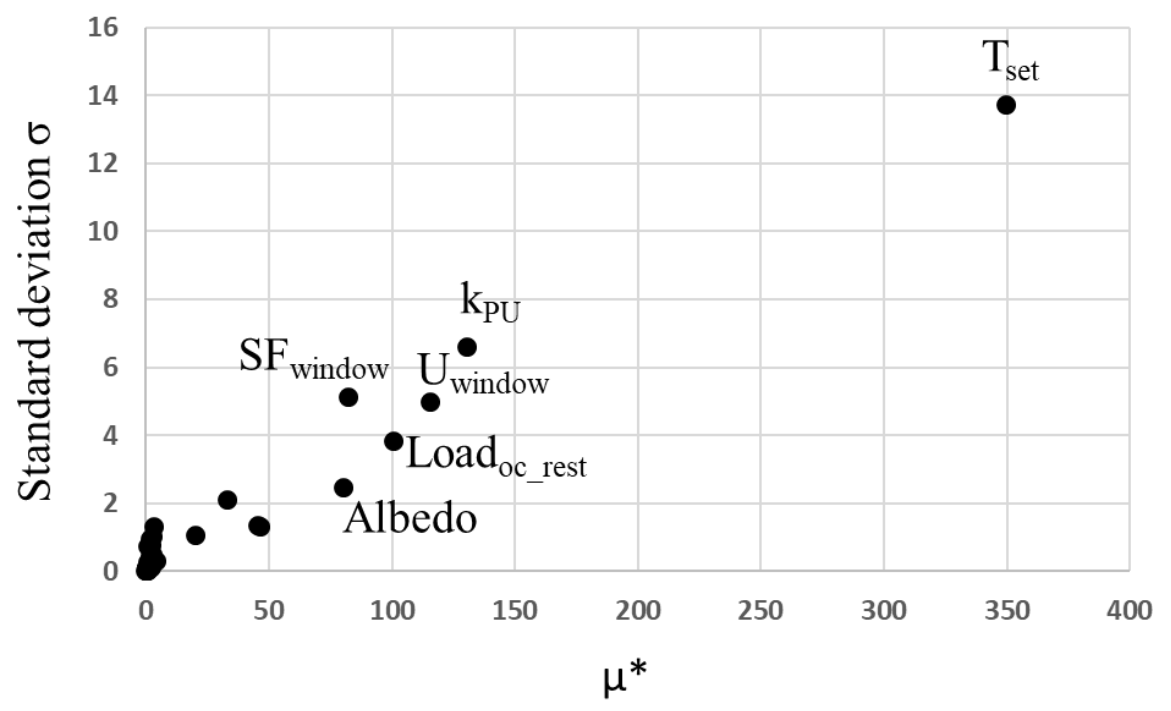

Figure 5 : Morris' Graph for the annual energy needs: the mean $\mu^{*}$ of the absolute effects for each parameter $(r=8)$.

Table 2: Sensitivity measure on annual heating energy needs and variation of the most influential parameters obtained with EASI RBD-FAST and Morris methods.

\begin{tabular}{|clccc|cc|}
\hline & \multicolumn{2}{c}{ Sensitivity analysis inputs } & \multicolumn{4}{c|}{ Sensitivity measure } \\
\hline \multirow{2}{*}{ Parameter } & $\begin{array}{c}\text { Variation of } \\
\text { the } \\
\end{array}$ & EASI RBD- & FAST & Morris & Rank \\
& & parameter & $\mathrm{S}_{\mathrm{i}}$ & $\mu^{*}$ & $\sigma$ & Morris \\
\hline $\mathrm{T}_{\text {set }}$ & Set-point temperature $\left[{ }^{\circ} \mathrm{C}\right]$ & $20 \pm 1$ & $61 \%$ & 350 & 14 & 1 \\
\hline $\mathrm{k}_{\mathrm{PU}}$ & Polyurethane thermal conductivity $\left[\mathrm{W} /\left(\mathrm{m}^{2} . \mathrm{K}\right)\right]$ & $\pm 10 \%$ & $14 \%$ & 130 & 7 & 2 \\
\hline $\mathrm{U}_{\text {window }}$ & Window thermal transmittance $\left[\mathrm{W} /\left(\mathrm{m}^{2} . \mathrm{K}\right)\right]$ & $\pm 10 \%$ & $8 \%$ & 116 & 5 & 3 \\
\hline Load $_{\text {oc_rest }}$ & Internal load - occupant at rest $[\mathrm{W}]$ & $63 \pm 10 \%$ & $<4 \%$ & 101 & 4 & 4 \\
\hline $\mathrm{SF}_{\text {window }}$ & Window solar factor $[-]$ & $\pm 10 \%$ & $<4 \%$ & 82 & 5 & 5 \\
\hline Albedo & Albedo $[-]$ & $0,3 \pm 0,1$ & $<4 \%$ & 80 & 2 & 6 \\
\hline
\end{tabular}

Note that the parameter sets identified as the most influential are the same with both methods. The set-point temperature is particularly influential, and the other variables are all in a zone of lower influence, which is found with both EASI RBD-FAST and the Morris method. EASI RBD-FAST allows in addition to quantify the impact of each parameter variability on the output variance; thus $61 \%$ of the dispersion is due to the set point temperature.

\subsubsection{Summer behaviour: Overheating risks on operative temperature}

The results for summer overheating in bedroom 1 are presented in Figure 6 and Table 3 of course, the heating set-point temperature is no longer one of the influencing parameters. The influential inputs are related to the cooling of the building with the night mechanical ventilation and the management of the heat contributions (solar factor, albedo, occupant). 


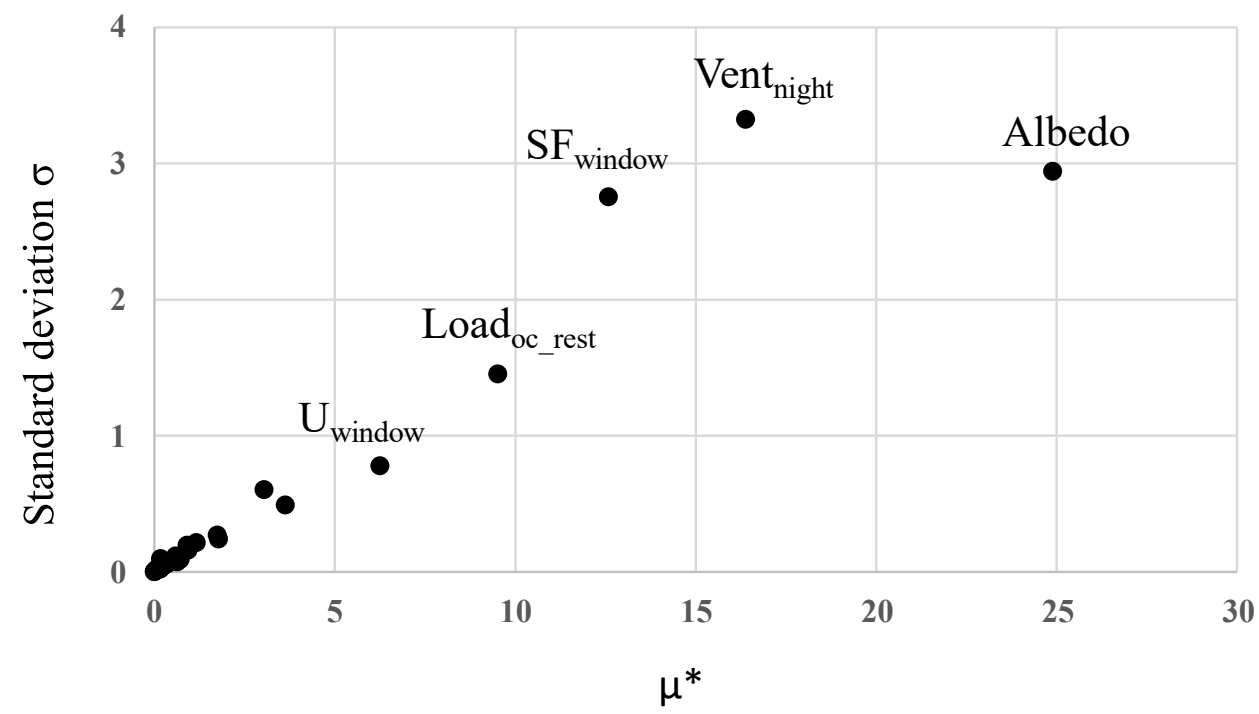

Figure 6 :Morris' Graph for the overheating risk: the mean $\mu^{*}$ of the absolute effects according to the standard deviation of the effects for each parameter $(r=8)$.

Table 3 : Sensitivity measure on overheating risks and variation of the parameters in the case of the most influential ones with EASI RBD-FAST and Morris.

\begin{tabular}{|clccc|cc|}
\hline & \multicolumn{3}{c}{ Sensitivity analysis inputs } & \multicolumn{3}{c|}{ Sensitivity measure } \\
\hline \multirow{2}{*}{ Parameter } & $\begin{array}{c}\text { Variation of } \\
\text { the parameter }\end{array}$ & $\begin{array}{c}\text { EASI RBD- } \\
\text { FAST }\end{array}$ & Morris & \multicolumn{2}{c|}{ Rank } \\
& & & $\mathrm{S}_{\mathrm{i}}$ & $\mu^{*}$ & $\sigma$ & Morris \\
\hline Albedo & Albedo [-] & $0,3 \pm 0,1$ & $45 \%$ & 25 & 3 & 1 \\
\hline Load $_{\text {oc_rest }}$ & Internal load - occupant at rest $[\mathrm{W}]$ & $63 \pm 10 \%$ & $14 \%$ & 10 & 1 & 4 \\
\hline Vent $_{\text {night }}$ & Night mechanical ventilation rate $[\mathrm{m} 3 / \mathrm{h}]$ & $\pm 20 \%$ & $13 \%$ & 16 & 3 & 2 \\
\hline $\mathrm{SF}_{\text {window }}$ & Window solar factor $[-]$ & $\pm 10 \%$ & $7 \%$ & 13 & 3 & 3 \\
\hline
\end{tabular}

In contrast to the results on winter behaviour, there is a discrepancy in ranking here between the Morris method and EASI RBD-FAST. Indeed, albedo is the most influential parameter for both methods but for the position of the next three, some permutations in the ranking between Morris and EASI RBDFAST methods are found.

\subsubsection{Discussion on sensitivity measure on aggregated indicator}

A comparison was made with Morris and EASI RBD-FAST on two aggregate indicators representative of building behaviour. The first step shows that on the same case with the same number of simulations, EASI RBD-FAST and Morris allow to obtain the same conclusions on the most influential inputs on the behaviour of the building. Although the ranking is slightly different between EASI RBD-FAST and Morris, both methods identify the same clusters of influential inputs which is still in line with the use of the Morris method as a screening method $[20,46]$. A difference in ranking is visible in Table 3 (summer behavior, see for example the rank of 'Load')) and can be explained by the increase in complexity and in particular the non-linearity of the phenomena involved in comparison with the winter behavior and which is investigated in section "3.2.2 Trends". The greater the complexity of the model, the more the ranking by Morris method is likely to be disturbed. However, it is not straightforward to assess the 
complexity of the model. This finding emphasizes the interest of using free model method as EASI RBD FAST, which means, that the method is suitable whatever the complexity of the model.

In addition, EASI RBD-FAST allows a quantification of each influence whereas the interpretation is sometimes ambiguous with Morris, especially upon considering an input as influential or not. It can also be tricky to know the amplitude of the influence gaps between inputs with the Morris method. For example, EASI RBD-FAST results show that albedo is 3 times more influential than internal loads.

From a physical interpretation point of view, the sensitivity analysis leads to the conclusion that the lack of knowledge about the inputs that regulate solar radiation through windows is what has the greatest impact on overheating periods. The management of inputs such as internal occupant loads, and night ventilation also have an impact. As a reminder, Bedroom 1 is occupied by two people at night unlike the other night rooms (one person only). It is important in a sensitivity analysis to clearly distinguish the nature of the different influencing parameters so that the impact on building performance can be reduced or not. In this case, it will be possible to intervene on the albedo and the night mechanical ventilation contrary to the internal loads and their variability.

The following section provides a more in-depth comparison of the amount of information extracted with RBD FAST

\subsection{Obtaining additional information with EASI RBD-FAST: uncertainty and trends}

\subsubsection{Output dispersion}

As recommended by Saltelli et al. in [1], it is always important to check that the output dispersion is significant to justify estimating the most influential parameters and therefore the sensitivity analysis. Unlike the Morris method, EASI RBD-FAST allows an uncertainty analysis in addition to the sensitivity analysis. In fact, the Morris method only considers uniform distributions, and the mesh and node method imply that the model is often evaluated across ranges of variations. This biases the output dispersion by overestimating the maximum and minimum values of the parameters as shown in Figure 7. Both input and output variability are represented in this figure for winter and summer behaviour and for both methods. 

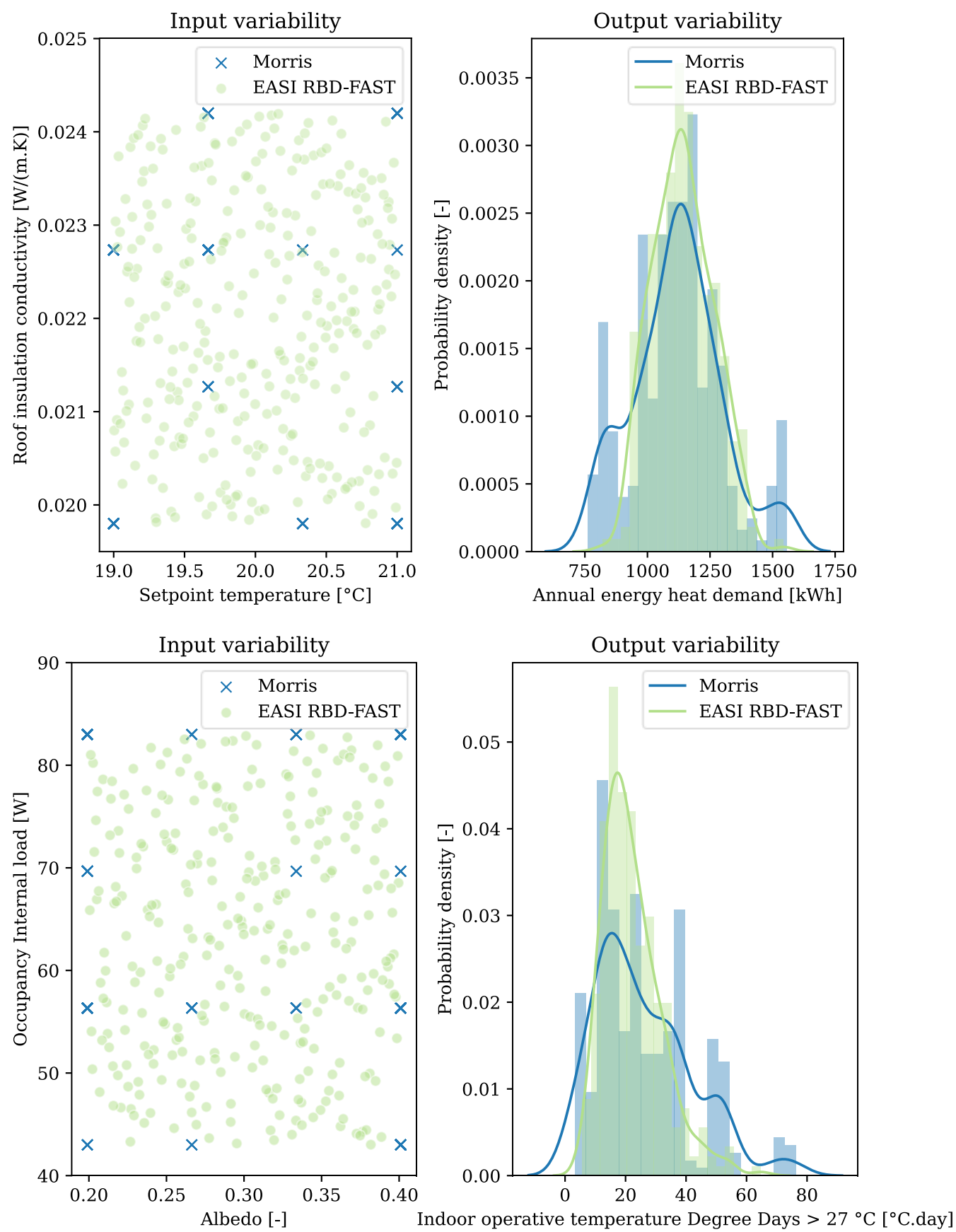

Figure 7: On the left the input variability is represented by the space filling for $\mathbf{2}$ influential parameters for each method and on the right the output variability is represented by the probability density of output of interest for each method. The upper side deals with the winter behaviour aggregate (annual energy needs), the lower side deals with the summer behaviour aggregate (overheating risks in the master bedroom).

The probability density heating demand distributions for the 312 simulations for each method are shown. The first observation concerns the dispersions of the two outputs that are significant, which justifies a sensitivity analysis to estimate the inputs responsible for the observed variability.

Discrepancy between both methods and the overestimation of the dispersion of heating demand with the Morris method can be observed. This difference is related to the method itself, that gives more importance to the points located on the boundaries of the parameter space. 
For the two outputs studied, we can clearly see on the graphs on the left the over-representation of the points located on the boundaries in the sampling according to Morris method. The impact of this over-representation of boundaries is shown in the Figure 7 on the right, where the higher occurrence of extreme values (outliers) for Morris compared to EASI RBD-FAST is visualized. This is particularly noticeable in the case of the heating demand (upper right side), where two peaks of occurrences can be seen at the minimum and maximum heating demand values.

This illustrates why Morris method results cannot be used for uncertainty analysis. It should be noted that for the heating demand, it can be observed that this spread of the probability density agrees with the variance and mean values obtained for each method as shown in Figure 4. The variance is indeed higher for Morris method due to larger spread.

\subsubsection{Trends}

Because the variance-based methods such as EASI RBD-FAST, allows uncertainty analysis, additional information about the main trends may be obtained using a simple scatterplot. In our study these are the heating need according to the set-point temperature, represented in Figure 8 , and the overheating degree.days of the main bedroom according to albedo and to mechanical night ventilation airflow shown in Figure 9.

The main observation is the difference in linearity between winter and summer behaviours. The heating need evolve linearly according to the value of the set-point temperature, and the observed dispersion remains constant whatever the temperature. For the summer behaviour, we observe that for the two most influential inputs: albedo on one hand (on the left) and the night mechanical ventilation on the other hand, the dispersion of the overheating depends on the value of the input. Dispersion is high for high albedo values as well as for low ventilation rate values. An interaction effect between these two inputs on the model output can be assumed.

To confirm this hypothesis, Figure 9-b shows, by colour level, the value of the overheating as a function of the albedo and mechanical night ventilation flow rate values. For ease of interpretation, an average coloration per hexagon is added so as not to give too much weight to a single simulation. It is clearly visualized that the overheating values for large folds occur at high albedo values and low night-time mechanical ventilation rates. From a physical interpretation point of view, it seems logical that the greater the solar contributions brought by the albedo, the more they will impact overheating, especially if the night cooling is low. This type of graphical representation makes it easier to understand and quantify it. However, the use of an annual aggregate of overheating risks limits the understanding of the phenomena and associated time constants as well as the temporal ranges of influence of the responsible inputs. Evaluating the influence of the inputs over a time-varying output would provide valuable insight: an additional method of analysis is therefore proposed in the following section. 


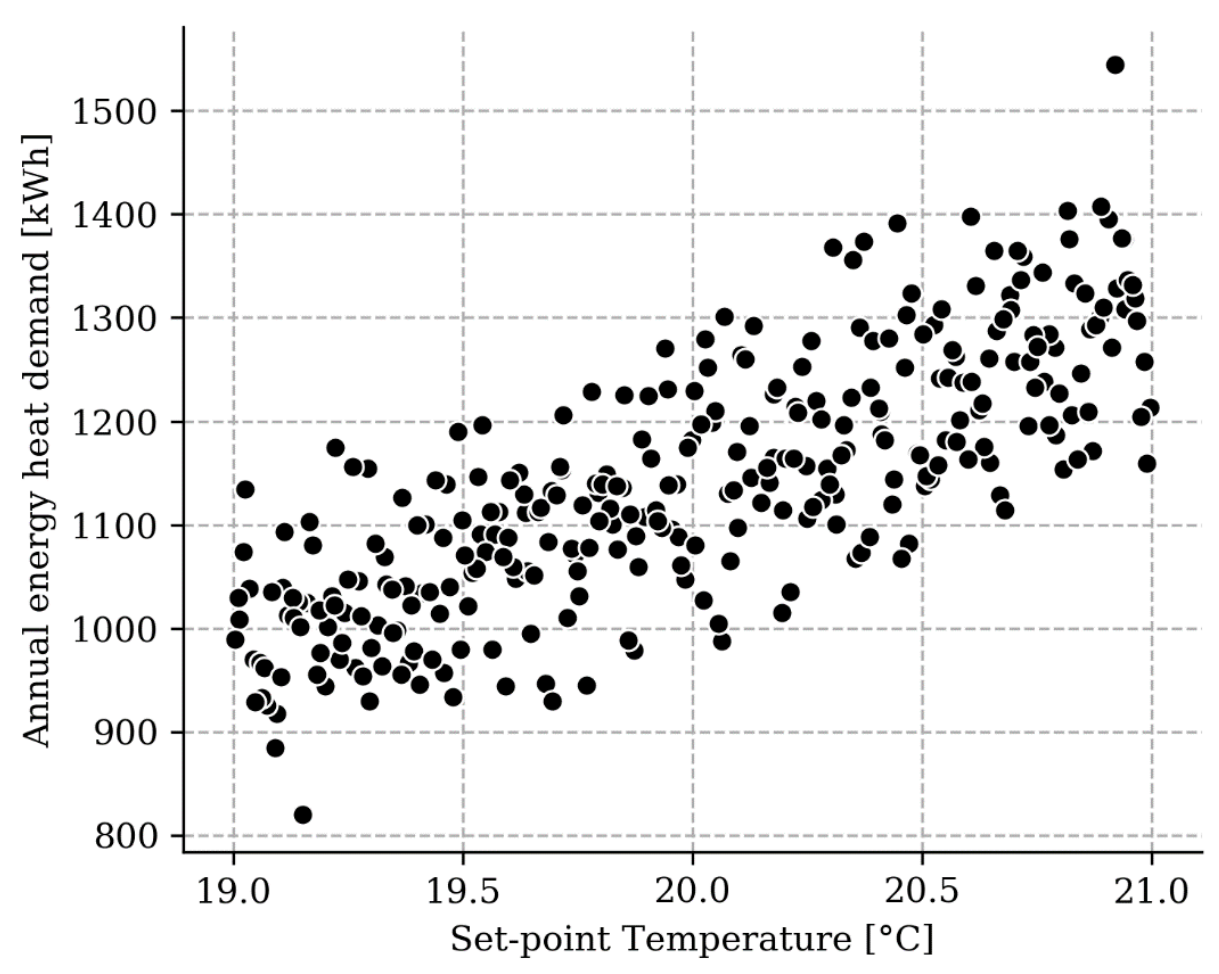

Figure 8 : Annual energy heating demand dispersion according to the set-point temperature variability from the 312 simulations performed with EASI RBD-FAST 
(a)
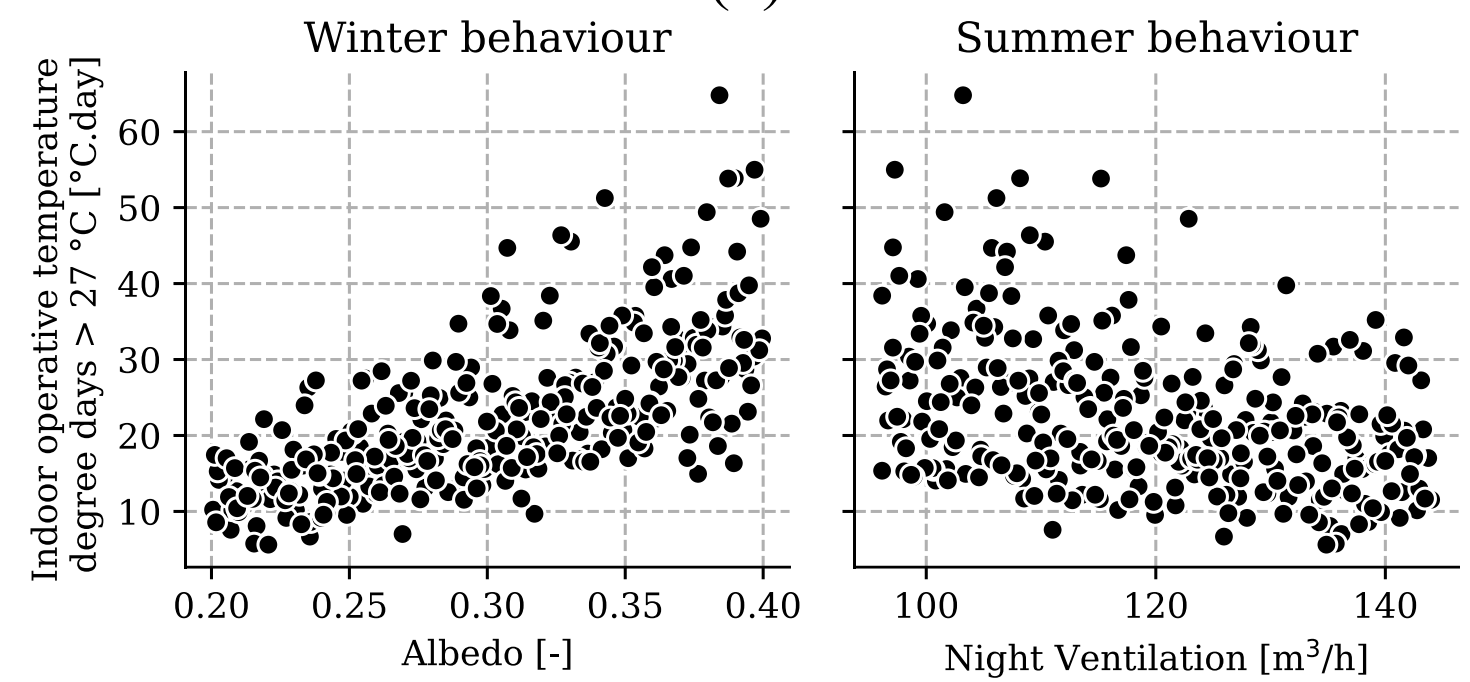

(b)

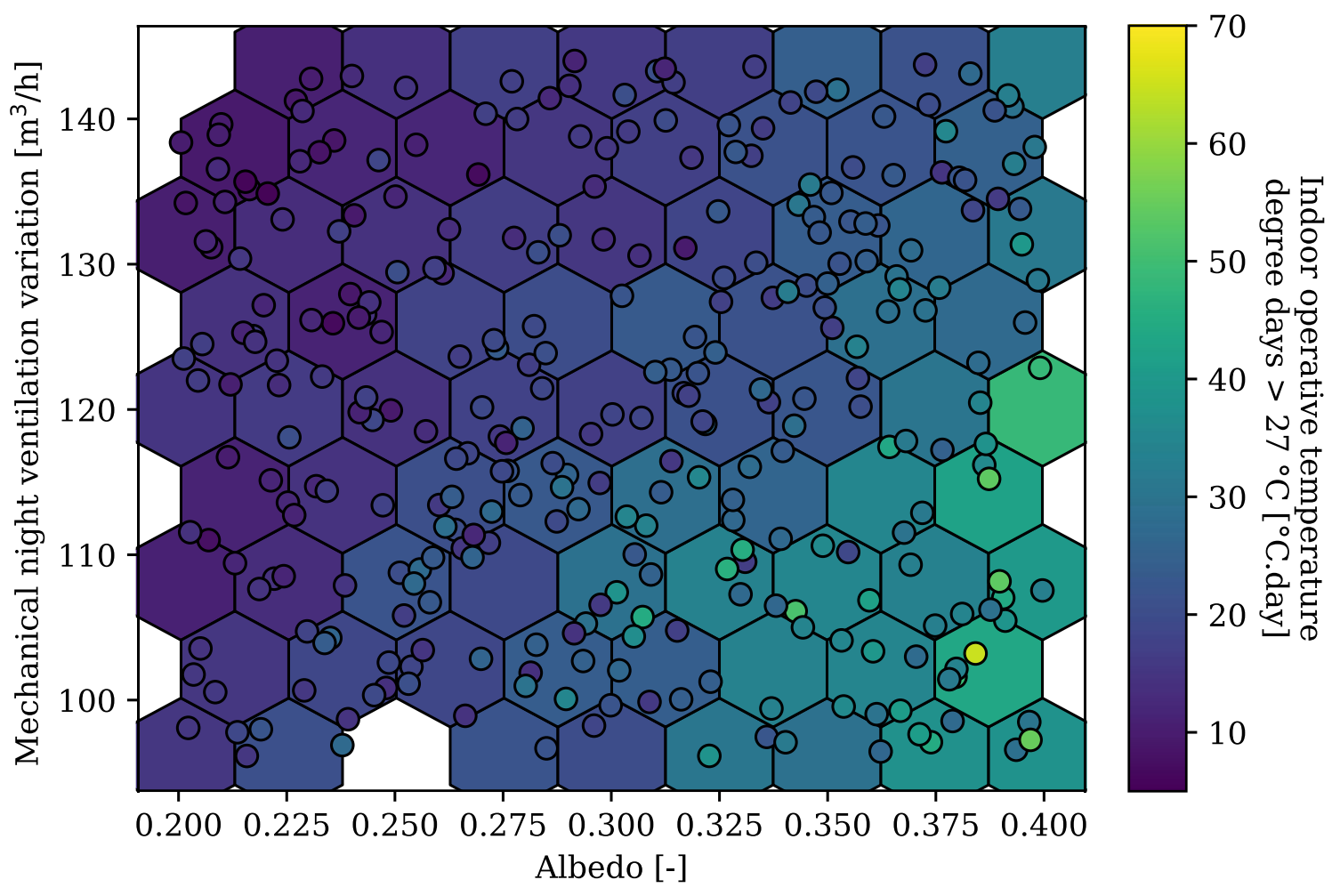

Figure 9 : On the top (a), overheating degree days dispersion according to albedo (on the left), and according to the night mechanical ventilation (on the right) from the 312 simulations performed with EASI RBD-FAST. On the lower side (b), graphical representation of the dependence of the output dispersion according to the albedo and the night mechanical ventilation values

\section{Global sensitivity analysis of time-dependent outputs using EASI RBD-FAST: future challenges}

This section explores the richness of the time-series information from the 312 simulations performed by EnergyPlus for the EASI RBD-FAST sensitivity analysis. Especially, and contrary to the 
Morris method, it is possible to go further than the aggregated outputs presented in the previous section by computing and visualizing at each timestep the output uncertainty and the sensitivity indexes. As shown in section 3.2 the OAT sampling design of the Morris sensitivity analysis does not allow the study of the output uncertainty.

In order to study the behaviour of the building on a finer time scale and in particular the summer behaviour, we take as the output of interest the operative temperature of Bedroom 1 at each hourly time step. First of all, the temperature variability is studied through the 312 simulations made with EASI RBD-FAST. Figure 10 shows the hourly evolution of the operative temperature throughout the year as well as its dispersion. The median value as well as the ranges of variation at $95 \%$ information are shown on the top graph. The lower graph shows the evolution of the standard deviation over time. For clarity, a Gaussian filter is applied to make the hourly variability less visible.

The observed temperature variability is not negligible and can reach at certain times of the year and for $95 \%$ of the configurations tested: \pm 1.2 degrees. It is observed that the operative temperature in summer between June and July reaches values $>30^{\circ} \mathrm{C}$ and that this coincides with the most significant standard deviation.
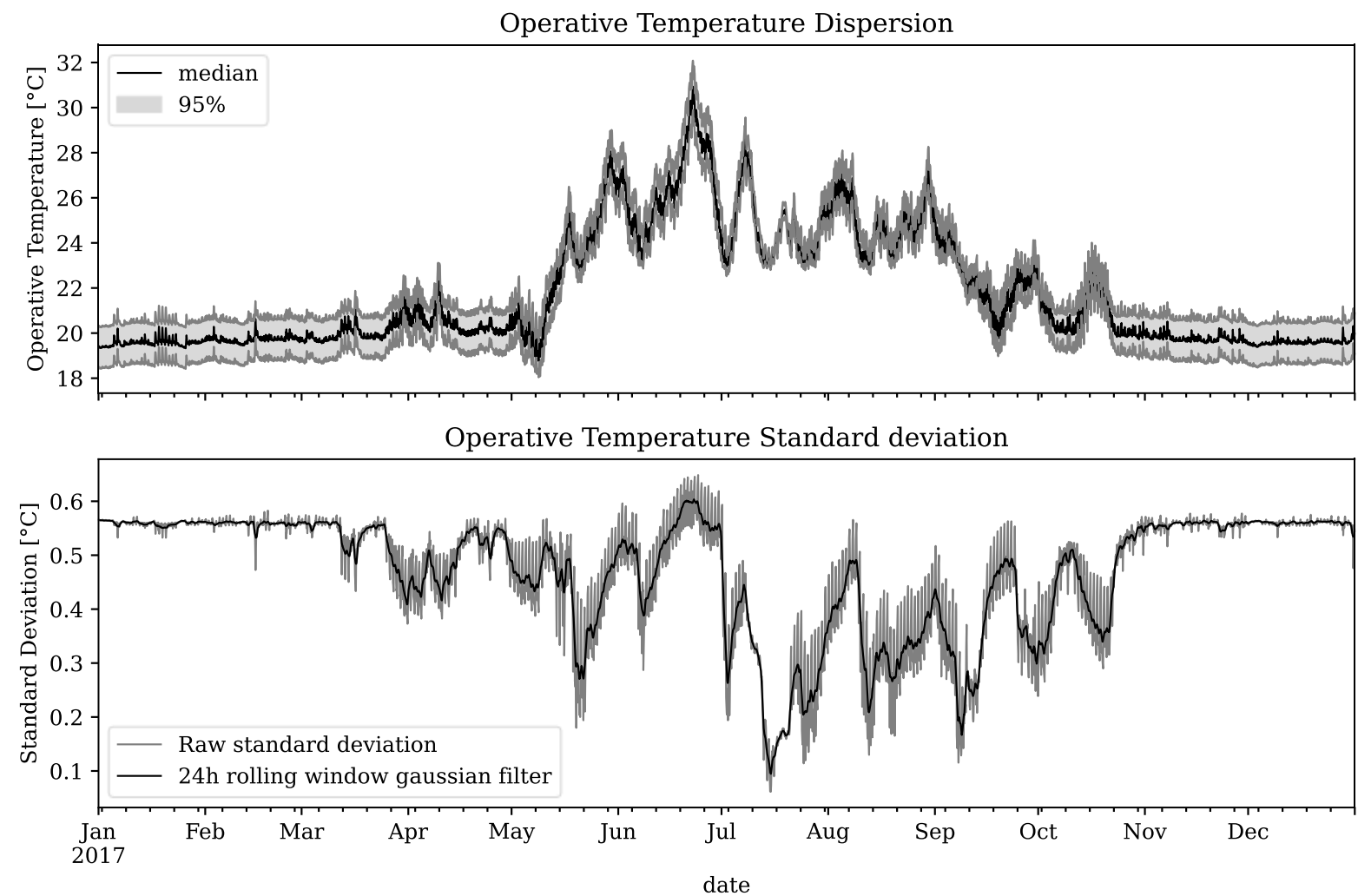

Figure 10 : Dispersion of the indoor operative temperature of the main bedroom with on the top the median value and the confidence bound at $95 \%$, on the bottom the standard deviation with the raw signal and a $24 \mathrm{~h}$ rolling window gaussian filter

In addition, there are two operating modes, one during the heating period, when the operative temperature remains constant, and one during the free evolution. During the latter period, the evolution of the uncertainty is variable over the whole period, as well as over a day when oscillations are observed (Figure 10 bottom graph). During the heating period, the uncertainty remains almost constant and appears stable also on an hourly scale. In order to understand which parameter is 
responsible for this change in dynamics on global and daily scales, sensitivity indices are computed at each time step and shown in Figure 11
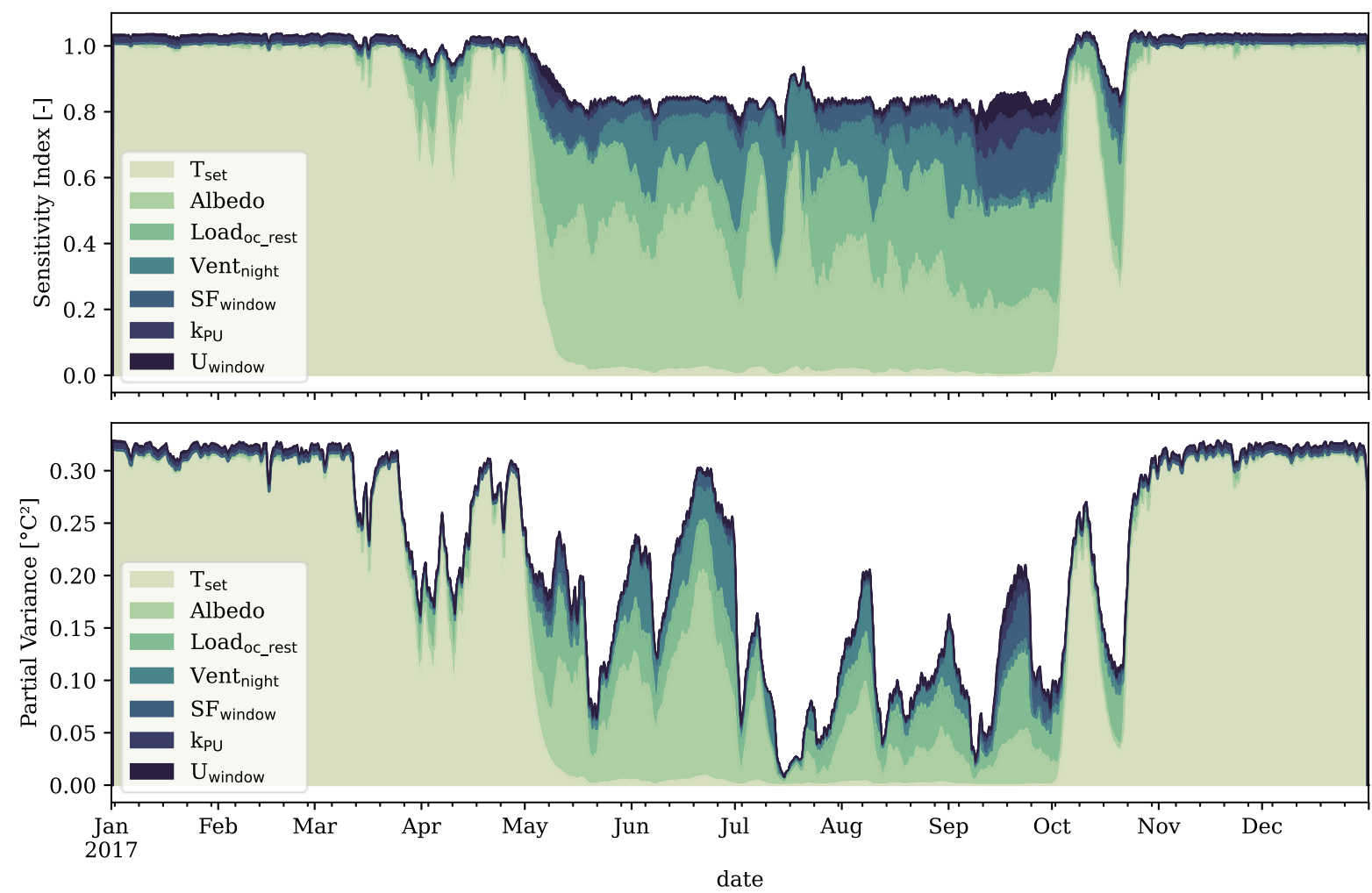

Figure 11 : Sensitivity index (on the top) and partial variance (on the bottom) of the indoor operative temperature of the main bedroom. For a better visualization, a gaussian filter with a $24 \mathrm{~h}$ window is applied on the profiles.

Figure 11 gathers the results on the EASI RBD-FAST sensitivity analysis of the bedroom operative temperature at each hourly timestep. The graph on the top represents the normalized sensitivity index, varying between 0 and 1 , and the bottom graph presents the partial variance for each input. These two results are complementary: the normalized sensitivity index is the partial variance divided by the total variance at each timestep. The advantage of partial variances is to visualize the variability of the total variance and the contribution of each input through the time.

Unsurprisingly, during the heating period, the variability in the operative temperature is only explained by the set-point temperature. The observed variability corresponds to the $\pm 1^{\circ} \mathrm{C}$ variation imposed on the setpoint temperature. Outside the heating period, during warmer days, variability of the operative temperature is intermittently influenced by a few parameters, and mainly by the albedo. The graph of classical sensitivity indices shows that there are significant interactions between the different parameters since the sum is well below 1 (sum to about $80 \%$ ). With the partial variance graph, it is possible to see the contribution of each input to the variance and thus the variability of the 312 simulations. In the summer period, several peaks of albedo influence are observed. Conversely, the comparison of both graphs shows how normalization through the calculation of the sensitivity indices hides zones where the variance is not very significant. For example, there is a holiday period in the simulation scenario (between July 1st and July $8^{\text {th }}$ ), which implies closed shutters and absence of the occupants. With the normalized sensitivity indices, this drop-in variance due to very different building management is not visualized, whereas the partial variance graph makes it very clear that the variability in the operative temperature is actually very low. Periods with low variance in the output 
are therefore not of great interest in a temporal analysis, as during this period the building 'absorbs' the variability of inputs. Consequently, the use of normalised sensitivity indexes is not appropriate for proper analysis of corresponding periods as they somehow artificially amplify the significance of inputs. From Figure 10 , it can be seen that periods above $27^{\circ} \mathrm{C}$ are quite rare, since we are interested in overheating, it is relevant to zoom in on the week when the building experiences the greatest overheating, i.e. the week of June $18-25,2017$. This allows to study in a more detailed way what impacts the variability of the high operative temperature for this period. Moreover, this period corresponds to the high variability of standard deviation seen on the bottom graph on Figure 10.

The evolution of the most influential inputs partial variances during the warmest week is plotted in the top of Figure 12. In the bottom, boundary conditions (outdoor temperature and direct solar radiation) are also plotted.
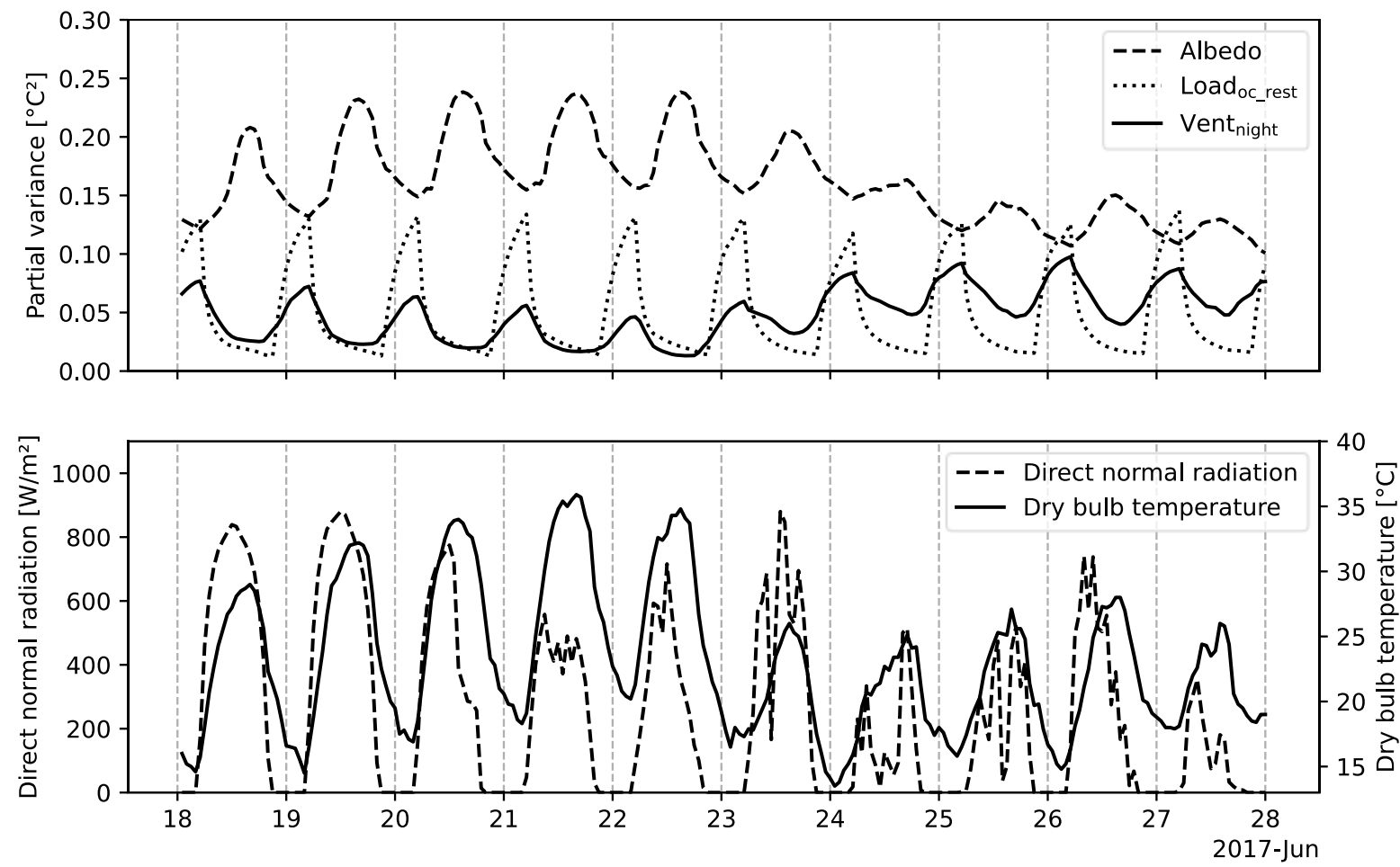

Figure 12 : On the top: Sensitivity measure with partial variance on the operative temperature of the main bedroom focused on one-week summer overheating. On the bottom, the corresponding weather conditions.

When looking at the sensitivity graph, the 3 most influential inputs are in order of importance: albedo, internal load due to occupants at rest and night mechanical ventilation rate. The difficulty of interpretation lies in the intermittent nature of these variables. It is important to differentiate the 'intervention' of the parameter in the physical behaviour of the building and its 'effect'. For example, the albedo intervenes in the building during the day. Its impact is certainly more important during the day but it still remains impacting at night. This persistent effect is explained by the inertia of the building. On the opposite, the Internal load - occupant at rest only comes into play at night and its influence decreases sharply during the day until it is no longer influential.

In the case of the night mechanical ventilation rate, we observe both a period with and without persistent influence during the day. The split of behaviour occurs on July 23d and 24th when a temperature and solar irradiation drop is observed. These changes in the outdoor weather conditions are concomitant to a decrease in the influence of the albedo and to an increase in the influence of the 
night ventilation. From this illustration can be understood how this approach allows a better understanding of the dynamic behaviour of the building, considering the climatic conditions.

As a partial conclusion, this section has shown the possibilities of the EASI RBD-FAST method for visualisation and understanding of building dynamics in the case of time dependent outputs such as the indoor temperature. On one hand, the visualization of the evolution of the uncertainty of the operating temperature over time shows the periods when the building is more robust - it 'resists' to input perturbations. In addition, this visualisation leads to quick identification of the overheating periods. On the other hand, the evolution of the sensitivity indices over time identifies those responsible for high uncertainty. The use of the partial variance brings in a single graph an easier interpretation of the results by combining the information on the evolution of the output uncertainty and the contribution of each responsible input. This visualization helps to expose the behaviour of the building as it varies according to seasons (i.e. winter, summer, spring/fall). A special focus is made on the highest overheating risk period that, in the present case study, is concomitant with the highest output uncertainty. High output uncertainty is precisely the most relevant to study, because it allows a possibility of reducing the temperature and therefore a possible leeway by the management of inputs and their uncertainty. However, it is important to identify which uncertainties are reducible, which are not and which management or change in the building may involve a decrease in the input effects. Some uncertainties can be minimized by on-site measurement or the input effect can be limited by building management such as albedo and the good use of shutters during the day for avoiding overheating. Nevertheless, other effects cannot be avoided, such as the heat load due to occupants during night. However, it is essential to consider their uncertainty and reduce it, if relevant, through additional information.

It should be noted that the variability observed for the output time series (here the operative indoor temperature) at an instant $t$ contains the impact of the variability of the inputs at this instant $t$ but also the impact of the variability of these inputs at the previous instants ( $t-1, t-2$, etc.). Consequently, when the sensitivity indices are estimated at an instant $t$, one part of the effects is due to simultaneous influence and another part to the effect of the inputs at previous time steps if these inputs are associated with inertial phenomena. This is the reason why, depending on the type of phenomenon involved, inertia and inertial effect can be assessed, as described previously. The information obtained through temporal sensitivity indices is therefore the identification and quantification of the effect of the inputs responsible for the uncertainty observed at time $t$ and depending on previous time steps. Such assessment of inertial phenomena is the stake of this study. However, advanced methods that build a surrogate model of the temporal output are able to separate the components of each instant and particularly to identify the inputs responsible for the features of the temporal dynamics $[47,48]$.

It should be noted, that the whole stake of a sensitivity and uncertainty analysis is in the very definition of the object of this study as specified in the steps set out by Lilburne and Tarantola in [49] for the implementation of a sensitivity and uncertainty analysis. This means that the results obtained in this paper regarding the building thermal behaviour are specific to the case study, the modelling choices, the underlying assumptions, the climate, etc. Nevertheless, the EASI RBD-FAST analysis of this case study illustrates the quality and the quantity of information that can be extracted, provided, naturally, that the convergence is reached with a sufficient number of simulations, here around 300 . Let us also recall that the choice of inputs as well as their associated probability distribution is known to influence the outcomes of any sensitivity analysis. The EASI RBD-FAST method remains valid with many types of probability distributions which is an asset for BPS studies. 


\section{CONCLUSION}

To encourage good practices in the use of sensitivity analysis and global uncertainty in BPS, the method used must be adapted to the building model, reliable, inexpensive in calculations, and, above all, accessible in terms of understanding and implementation [11]. In the present article we showed that the EASI RBD-FAST method, easily accessible by its recent implementation in SA Lib (python sensitivity analysis toolbox), combines the efficiency and adaptability of variance-based methods while maintaining a competitive simulation cost. EASI RBD-FAST has been compared to the widely used Morris screening method on two annual outputs representative of winter and summer behaviour of a building.

Our results show that both methods: classically used Morris method and more recent EASI RBD-FAST variance-based method fulfil basic expectations, since the same clusters of influential parameters were identified. However, while deepening the results analyses some differences were found. The behaviour of the building energy model in summer was found non-linear and the Morris-ranking of influential inputs was slightly different compared with the variance-based analysis obtained with EASI RBD-FAST. This agrees with the work of Menberg et al. [20], Kristensen and Petersen [46], and with the commonly acknowledged good practices in sensitivity analysis [10]. Our results demonstrate that the Morris method is indeed useful as a first-step analysis to identify the most influential parameters, but it is recommended to supplement it by a variance-based analysis which then quantifies the influence of each input parameter and associates an uncertainty analysis.

We have also shown that the EASI RBD-FAST method allows, with the same number of simulations, to extract more information, such as quantified sensitivity indices, uncertainty analysis, visualising interactions and correlations between the inputs and the outputs. Moreover, EASI RBD-FAST, is applicable regardless of the model and its complexity; it is therefore flexible and adjustable to any BPS study: temporal sensitivity indices, thermal discomfort thresholds, etc, without additional computational cost.

The visualisation of the temporal uncertainty and the temporal partial variance of each input leads to emphasize relevant period of study with respect to dynamic properties of the building such as persistence or inertia. This is a novel and very promising application of SA/UA, for example to mitigate summer overheating problems, as proposed by Gondian et $a l$. [50].

To sum it all up, the EASI RBD-FAST method illustrated step-by-step in this paper proves to be a useful and accessible tool for both the researcher and the practitioner. It offers appealing possibilities to comprehensively study the behaviour of a building energy model for various purposes and at different time scales. We hope that the present paper will contribute to the development of good practices for UA/SA in building performance assessment. This work is part of a larger project aiming at encouraging good practice in sensitivity and uncertainty analysis by the use of the EASI RBD-FAST method. The project itself has been the opportunity to implement EASI RBD-FAST analysis in the Python package SALib [45] and to disseminate these methods through international workshops and communications [51]. 


\section{ACKNOWLEDGEMENTS}

The authors would like to thank the French Environment \& Energy Management Agency (ADEME) for funding this research through the OLIMP Project. The authors would also like to thank Sarah Juricic for her valuable advice and proofreading, and Nicolas Cellier for his help in improving data visualisation in Python.

\section{REFERENCES}

[1] A. Saltelli, K. Aleksankina, W. Becker, P. Fennell, F. Ferretti, N. Holst, S. Li, Q. Wu, Why so many published sensitivity analyses are false: A systematic review of sensitivity analysis practices, $\begin{array}{llllll}\text { Environmental Modelling } \quad \& \quad \text { Software. } & 114 & \text { (2019) }\end{array}$ https://doi.org/10.1016/j.envsoft.2019.01.012.

[2] G.A. Faggianelli, L. Mora, R. Merheb, Uncertainty quantification for Energy Savings Performance Contracting: Application to an office building, Energy and Buildings. 152 (2017) 61-72. https://doi.org/10.1016/j.enbuild.2017.07.022.

[3] A. Chong, K. Menberg, Guidelines for the Bayesian calibration of building energy models, Energy and Buildings. 174 (2018) 527-547. https://doi.org/10.1016/j.enbuild.2018.06.028.

[4] M. Senave, S. Roels, S. Verbeke, D. Saelens, Analysis of the influence of the definition of the interior dwelling temperature on the characterization of the heat loss coefficient via on-board monitoring, Energy and 109860. https://doi.org/10.1016/j.enbuild.2020.109860.

[5] T. Østergård, R.L. Jensen, S.E. Maagaard, Early Building Design: Informed decision-making by exploring multidimensional design space using sensitivity analysis, Energy and Buildings. 142 (2017) 8-22. https://doi.org/10.1016/j.enbuild.2017.02.059.

[6] B. Raji, M.J. Tenpierik, A. van den Dobbelsteen, An assessment of energy-saving solutions for the envelope design of high-rise buildings in temperate climates: A case study in the Netherlands, Energy and Buildings. 124 (2016) 210-221. https://doi.org/10.1016/j.enbuild.2015.10.049.

[7] F. Monari, P. Strachan, Characterization of an airflow network model by sensitivity analysis: parameter screening, fixing, prioritizing and mapping, Journal of Building Performance Simulation. 10 (2017) 17-36. https://doi.org/10.1080/19401493.2015.1110621.

[8] T.A. Mara, S. Tarantola, Application of global sensitivity analysis of model output to building thermal simulations, Build. Simul. 1 (2008) 290-302. https://doi.org/10.1007/s12273-008-81295.

[9] B. looss, P. Lemaître, A review on global sensitivity analysis methods, in: Uncertainty Management in Simulation-Optimization of Complex Systems, Springer, 2014: pp. 101-122. http://arxiv.org/abs/1404.2405 (accessed June 16, 2020).

[10] A. Saltelli, ed., Global sensitivity analysis: the primer, John Wiley, Chichester, England ; Hoboken, NJ, 2008.

[11] D. Douglas-Smith, T. Iwanaga, B.F.W. Croke, A.J. Jakeman, Certain trends in uncertainty and sensitivity analysis: An overview of software tools and techniques, Environmental Modelling \& Software. 124 (2020) 104588. https://doi.org/10.1016/j.envsoft.2019.104588.

[12] F. Ferretti, A. Saltelli, S. Tarantola, Trends in sensitivity analysis practice in the last decade, Science of The Total Environment. 568 (2016) 666-670. https://doi.org/10.1016/j.scitotenv.2016.02.133. 
[13] W. Tian, A review of sensitivity analysis methods in building energy analysis, Renewable and Sustainable Energy Reviews. 20 (2013) 411-419. https://doi.org/10.1016/j.rser.2012.12.014.

[14] Z. Pang, Z. O'Neill, Y. Li, F. Niu, The role of sensitivity analysis in the building performance analysis: A critical review, Energy and Buildings. $209 \quad$ (2020) 109659. https://doi.org/10.1016/j.enbuild.2019.109659.

[15] S. Petersen, M.H. Kristensen, M.D. Knudsen, Prerequisites for reliable sensitivity analysis of a high fidelity building energy model, Energy and Buildings. 183 (2019) 1-16. https://doi.org/10.1016/j.enbuild.2018.10.035.

[16] J.H. Kämpf, M. Wetter, D. Robinson, A comparison of global optimization algorithms with standard benchmark functions and real-world applications using EnergyPlus, Journal of Building Performance Simulation. 3 (2010) 103-120. https://doi.org/10.1080/19401490903494597.

[17] A.-T. Nguyen, S. Reiter, P. Rigo, A review on simulation-based optimization methods applied to building performance analysis, Applied Energy. 113 (2014) 1043-1058. https://doi.org/10.1016/j.apenergy.2013.08.061.

[18] A. Saltelli, P. Annoni, How to avoid a perfunctory sensitivity analysis, Environmental Modelling \& Software. 25 (2010) 1508-1517. https://doi.org/10.1016/j.envsoft.2010.04.012.

[19] I.M. Sobol', Sensitivity analysis for non-linear mathematical models, MathematicalModelling and Computational Experimen. 1 (1993) 407-414.

[20] K. Menberg, Y. Heo, R. Choudhary, Sensitivity analysis methods for building energy models: Comparing computational costs and extractable information, Energy and Buildings. 133 (2016) 433-445. https://doi.org/10.1016/j.enbuild.2016.10.005.

[21] A.-T. Nguyen, S. Reiter, A performance comparison of sensitivity analysis methods for building energy models, Build. Simul. 8 (2015) 651-664. https://doi.org/10.1007/s12273-015-0245-4.

[22] R. Confalonieri, G. Bellocchi, S. Bregaglio, M. Donatelli, M. Acutis, Comparison of sensitivity analysis techniques: A case study with the rice model WARM, Ecological Modelling. 221 (2010) 1897-1906. https://doi.org/10.1016/j.ecolmodel.2010.04.021.

[23] H.M. Wainwright, S. Finsterle, Q. Zhou, J.T. Birkholzer, Modeling the performance of large-scale $\mathrm{CO} 2$ storage systems: A comparison of different sensitivity analysis methods, International Journal of Greenhouse Gas Control. 17 (2013) 189-205. https://doi.org/10.1016/j.ijggc.2013.05.007.

[24] F. Campolongo, J. Cariboni, A. Saltelli, An effective screening design for sensitivity analysis of large models, Environmental Modelling \& Software. 22 (2007) 1509-1518. https://doi.org/10.1016/j.envsoft.2006.10.004.

[25] T. Østergård, S.E. Maagaard, R.L. Jensen, A STOCHASTIC AND HOLISTIC METHOD TO SUPPORT DECISION-MAKING IN EARLY BUILDING DESIGN, in: Hyderabad, India, 2015: p. 8.

[26] T. Alex Mara, O. Rakoto Joseph, Comparison of some efficient methods to evaluate the main effect of computer model factors, Journal of Statistical Computation and Simulation. 78 (2008) 167-178. https://doi.org/10.1080/10629360600964454.

[27] J. Goffart, T. Mara, E. Wurtz, Generation of stochastic weather data for uncertainty and sensitivity analysis of a low-energy building, Journal of Building Physics. 41 (2017) 41-57. https://doi.org/10.1177/1744259116668598.

[28] F. Anstett-Collin, J. Goffart, T. Mara, L. Denis-Vidal, Sensitivity analysis of complex models: Coping with dynamic and static inputs, Reliability Engineering \& System Safety. 134 (2015) 268-275. https://doi.org/10.1016/j.ress.2014.08.010. 
[29] A. Saltelli, P. Annoni, I. Azzini, F. Campolongo, M. Ratto, S. Tarantola, Variance based sensitivity analysis of model output. Design and estimator for the total sensitivity index, Computer Physics Communications. 181 (2010) 259-270. https://doi.org/10.1016/j.cpc.2009.09.018.

[30] S. Tarantola, D. Gatelli, T.A. Mara, Random balance designs for the estimation of first order global sensitivity indices, Reliability Engineering \& System Safety. 91 (2006) 717-727. https://doi.org/10.1016/j.ress.2005.06.003.

[31] E. Plischke, An effective algorithm for computing global sensitivity indices (EASI), Reliability Engineering \& System Safety. 95 (2010) 354-360. https://doi.org/10.1016/j.ress.2009.11.005.

[32] A.P. de A. Rocha, J. Goffart, L. Houben, N. Mendes, On the uncertainty assessment of incident direct solar radiation on building facades due to shading devices, Energy and Buildings. 133 (2016) 295-304. https://doi.org/10.1016/j.enbuild.2016.09.058.

[33] J. Goffart, M. Rabouille, N. Mendes, Uncertainty and sensitivity analysis applied to hygrothermal simulation of a brick building in a hot and humid climate, Journal of Building Performance Simulation. 10 (2017) 37-57. https://doi.org/10.1080/19401493.2015.1112430.

[34] I.M. Sobol', Global sensitivity indices for nonlinear mathematical models and their Monte Carlo estimates, Mathematics and Computers in Simulation. 55 (2001) 271-280. https://doi.org/10.1016/S0378-4754(00)00270-6.

[35] A. Saltelli, R. Bolado, An alternative way to compute Fourier amplitude sensitivity test (FAST), Computational Statistics \& Data Analysis. 26 (1998) 445-460. https://doi.org/10.1016/S01679473(97)00043-1.

[36] B. Sudret, Global sensitivity analysis using polynomial chaos expansions, Reliability Engineering \& System Safety. 93 (2008) 964-979. https://doi.org/10.1016/j.ress.2007.04.002.

[37] M. Ratto, A. Pagano, P. Young, State Dependent Parameter metamodelling and sensitivity analysis, Computer Physics Communications. $177 \quad$ (2007) 863-876. https://doi.org/10.1016/j.cpc.2007.07.011.

[38] J.-Y. Tissot, C. Prieur, Bias correction for the estimation of sensitivity indices based on random balance designs, Reliability Engineering \& System Safety. 107 (2012) 205-213. https://doi.org/10.1016/j.ress.2012.06.010.

[39] G. Damblin, M. Couplet, B. looss, Numerical studies of space-filling designs: optimization of Latin Hypercube Samples and subprojection properties, Journal of Simulation. 7 (2013) 276-289. https://doi.org/10.1057/jos.2013.16.

[40] J. Goffart, M. Woloszyn, X. Faure, F. Wurtz, L. Gondian, C. Buhé, T. Recht, L. Mora, B. Peuportier, P. Schalbart, M. Aymari, S. Ploix, P. Schneuwly, E. Wurtz, Overview Of A Large Scale Monitoring Project Of Energy Positive Houses: Complementarity Between Simulations And Measurements, in: 16th IBPSA Conference, 2019.

[41] L. Gondian, Application de l'analyse de sensibilité temporelle pour la description de la réponse thermique d'un bâtiment aux actions des habitants, Université Savoie Mont-Blanc, 2019.

[42] D.B. Crawley, L.K. Lawrie, F.C. Winkelmann, W.F. Buhl, Y.J. Huang, C.O. Pedersen, R.K. Strand, R.J. Liesen, D.E. Fisher, M.J. Witte, J. Glazer, EnergyPlus: creating a new-generation building energy simulation program, Energy and Buildings. 33 (2001) 319-331. https://doi.org/10.1016/S03787788(00)00114-6.

[43] W. Tian, Y. Heo, P. de Wilde, Z. Li, D. Yan, C.S. Park, X. Feng, G. Augenbroe, A review of uncertainty analysis in building energy assessment, Renewable and Sustainable Energy Reviews. 93 (2018) 285-301. https://doi.org/10.1016/j.rser.2018.05.029. 
[44] C. Spitz, L. Mora, E. Wurtz, A. Jay, Practical application of uncertainty analysis and sensitivity analysis on an experimental house, Energy and Buildings. 55 (2012) 459-470. https://doi.org/10.1016/j.enbuild.2012.08.013.

[45] J. Herman, W. Usher, SALib: An open-source Python library for Sensitivity Analysis, Journal of Open Source Software. 2 (2017) 97. https://doi.org/10.21105/joss.00097.

[46] M.H. Kristensen, S. Petersen, Choosing the appropriate sensitivity analysis method for building energy model-based investigations, Energy and Buildings. 130 (2016) 166-176. https://doi.org/10.1016/j.enbuild.2016.08.038.

[47] M. Lamboni, H. Monod, D. Makowski, Multivariate sensitivity analysis to measure global contribution of input factors in dynamic models, Reliability Engineering \& System Safety. 96 (2011) 450-459. https://doi.org/10.1016/j.ress.2010.12.002.

[48] K. Campbell, M.D. McKay, B.J. Williams, Sensitivity analysis when model outputs are functions, Reliability Engineering \& System Safety. $91 \quad$ (2006) 1468-1472. https://doi.org/10.1016/j.ress.2005.11.049.

[49] L. Lilburne, S. Tarantola, Sensitivity analysis of spatial models, International Journal of Geographical Information Science. $23 \quad$ (2009) 151-168. https://doi.org/10.1080/13658810802094995.

[50] L. Gondian, J. Goffart, M. Woloszyn, C. Buhé, P. Maréchal, E. Wurtz, Towards Assessing Houses Resistance and Resilience Indicators At Inhabitants' Actions Using Temporal Sensitivity Analysis, in: Rome, Italy, 2019: p. 8.

[51] J. Goffart, S. Juricic, N. Cellier, Uncertainty and Sensitivity Analysis, in: GitHub Repository, Aussois, 2018. https://github.com/locie/simurex2018_workshop/tree/master/goffartj/sensitivity (accessed February 1, 2021). 